\title{
Brightest galaxies as halo centre tracers in SDSS DR7
}

\author{
Johannes U. Lange ${ }^{1,2}$, Frank C. van den Bosch ${ }^{1}$, Andrew Hearin ${ }^{3}$, \\ Duncan Campbell ${ }^{1}$, Andrew R. Zentner ${ }^{4}$, Antonia Villarreal ${ }^{4}$, and \\ Yao-Yuan $\mathrm{Mao}^{4}$ \\ ${ }^{1}$ Department of Astronomy, Yale University, P.O. Box 208101, New Haven, CT \\ ${ }^{2}$ Kavli Institute for Theoretical Physics, University of California, Santa Barbara, CA \\ ${ }^{3}$ Yale Center for Astronomy $\&$ Astrophysics, Yale University, New Haven, CT \\ ${ }^{4}$ Department of Physics and Astronomy \& Pittsburgh Particle Physics, Astrophysics, and Cosmology Center (PITT PACC), \\ University of Pittsburgh, Pittsburgh, PA 15260
}

Accepted xxx. Received xxx

\begin{abstract}
Determining the positions of halo centres in large-scale structure surveys is crucial for many cosmological studies. A common assumption is that halo centres correspond to the location of their brightest member galaxies. In this paper, we study the dynamics of brightest galaxies with respect to other halo members in the Sloan Digital Sky Survey DR7. Specifically, we look at the line-of-sight velocity and spatial offsets between brightest galaxies and their neighbours. We compare those to detailed mock catalogues, constructed from high-resolution, dark-matter-only $N$-body simulations, in which it is assumed that satellite galaxies trace dark matter subhaloes. This allows us to place constraints on the fraction $f_{\mathrm{BNC}}$ of haloes in which the brightest galaxy is not the central. Compared to previous studies we explicitly take into account the unrelaxed state of the host haloes, velocity offsets of halo cores and correlations between $f_{\mathrm{BNC}}$ and the satellite occupation. We find that $f_{\mathrm{BNC}}$ strongly decreases with the luminosity of the brightest galaxy and increases with the mass of the host halo. Overall, in the halo mass range $10^{13}-10^{14.5} h^{-1} \mathrm{M}_{\odot}$ we find $f_{\mathrm{BNC}} \sim 30 \%$, in good agreement with a previous study by Skibba et al. We discuss the implications of these findings for studies inferring the galaxy-halo connection from satellite kinematics, models of the conditional luminosity function and galaxy formation in general.
\end{abstract}

Key words: methods: statistical - galaxies: kinematics and dynamics - galaxies: groups: general - cosmology: dark matter

\section{INTRODUCTION}

In the standard cosmological model, cold dark matter (CDM) haloes provide the potential wells that enable the condensation of gas into stars. Ultimately, this causes CDM halos to be the natural cites for the formation of galaxies (see Mo et al. 2010, for a review). Thus, galaxies and the dark matter haloes in which they reside constitute an inseparable entity.

Hierarchical structure formation is a fundamental prediction of the $\Lambda \mathrm{CDM}$ cosmological model. In this paradigm, small dark matter haloes form first and grow by the accretion of surrounding dark matter mass and other smaller haloes. Accreted dark matter haloes can survive inside the the host halo for many dynamical times. These accreted haloes are called subhaloes and they are associated with satellite galaxies. The galaxy harboured by the host halo is known as the central galaxy.

It is generally assumed that satellite galaxies accreted by a bigger halo are slowly quenched by processes unique to the cluster or group environment and stop forming new stars, whereas the central galaxy continues to grow. Additionally, once satellite galaxies are disrupted by gravitational interactions inside the host, it is assumed that part of their stellar material is accreted onto the central galaxy. Given this picture, it is expected that central galaxies form a distinctive population of galaxies that are systematically more massive and more luminous than their satellite galaxies. In fact, it is commonly assumed that the brightest galaxy inside a dark matter halo is often, if not always, the central galaxy.

Indeed, observations have shown that brightest halo galaxies (BHGs) form a distinctive population of halo mem- 
bers. Their luminosities cannot be predicted by defining them as the brightest galaxy out of a population whose luminosities are drawn from the same underlying distribution (e.g., Tremaine \& Richstone 1977; Lin et al. 2010; Hearin et al. 2013a; Shen et al. 2014). This can be interpreted as BHGs being more luminous because they are central galaxies of the halo. However, there is no firm reason to assume that BHGs always coincide with the centres of dark matter halos. For example, central galaxies could have spatial or velocity offsets from the actual halo centre. Additionally, it is expected that in a fraction $f_{\mathrm{BNC}}$ of the haloes the brightest (or most massive) galaxy is not the central, but a satellite galaxy. This has been analysed specifically for galaxy clusters (see, e.g. Sanderson et al. 2009; Zitrin et al. 2012; Hikage et al. 2013; Wang et al. 2014; Lauer et al. 2014; Hoshino et al. 2015) for which the large number of satellite galaxies and the bright X-ray emitting gas make it relatively easy to determine the halo centre. However, there are so far very few studies analysing the phase-space positions of BHGs in low-mass haloes.

Recently, Guo et al. (2015b) modelled the redshift-space clustering of galaxies in SDSS allowing centrals to have velocity offsets with respect to their dark matter haloes. Their results suggest that even in low-mass haloes central galaxies are not at rest relative to the dark matter. van den Bosch et al. (2005b) and Skibba et al. (2011) have analysed the phase-space coordinates of BHGs in SDSS, concluding that central galaxies are either not at rest with respect to the dark matter halo or some satellites are BHGs, i.e. $f_{\mathrm{BNC}} \neq 0$. More specifically, Skibba et al. (2011) provided evidence that the latter case is the main reason for the mis-centering of BHGs. They analysed $f_{\mathrm{BNC}}$ for a large range of halo masses down to $\sim 10^{12.5} h^{-1} M_{\odot}$ and found values of $f_{\mathrm{BNC}}$ that increase from $\sim 25 \%$ for Milky-Way size haloes to $\sim 45 \%$ for massive clusters. Interestingly, those values are higher than predicted by semi-analytical models (SAMs) of galaxy formation (Skibba et al. 2011). The authors argued that their finding could be interpreted as evidence for longer quenching and dynamical friction time-scales than commonly assumed in galaxy formation theory. However, the results of Skibba et al. (2011) are in tension with those of Hoshino et al. (2015), who find a significantly lower $f_{\mathrm{BNC}}$ of $\sim 20-30 \%$ for clusters.

In this work, we employ an analysis technique similar to the one used by Skibba et al. (2011). Specifically, we analyse offsets in phase-space between BHGs and other halo members. We improve upon the analysis of Skibba et al. (2011) by taking into account several effects that could potentially bias the inferred values of $f_{\mathrm{BNC}}$. These effects include velocity offsets of halo cores and central galaxies with respect to the main halo, halo triaxiality, the unrelaxed state of the satellite population and the correlation of $f_{\mathrm{BNC}}$ with satellite occupation. Additionally, we characterize how $f_{\mathrm{BNC}}$ depends on both halo mass $M$ and BHG luminosity $L_{\mathrm{BHG}}$ instead of halo mass alone. The dependence on $L_{\mathrm{BHG}}$ is particularly important because it is observationally more easily accessible than halo mass.

Determining $f_{\mathrm{BNC}}$ is crucial for many cosmological studies. For example, weak lensing surveys often explore gravitational lensing around brightest cluster galaxies (BCGs) (see, e.g. Sheldon et al. 2009a,b) or satellites (see, e.g. Yang et al. 2006; Li et al. 2013, 2014; Sifón et al. 2015; Li et al. 2016; Niemiec et al. 2017). Knowing $f_{\mathrm{BNC}}$ is necessary in this case to get an unbiased estimate of the corresponding dark matter halo mass (Johnston et al. 2007; Li et al. 2014). Additionally, modelling the (redshift-dependent) clustering of galaxies using halo occupation distribution (HOD) modelling implicitly requires knowledge of $f_{\mathrm{BNC}}$. For example, the average satellite occupation of dark matter halos is typically scaled by the average central occupation (Zheng et al. 2007; Zehavi et al. 2011; Leauthaud et al. 2012). Alternatively, assuming that only haloes that host a central galaxy can also host satellites above a given threshold (see, e.g. Guo et al. 2015b) is equivalent to assuming that $f_{\mathrm{BNC}}=0$. Furthermore, many studies of the kinematics of satellite galaxies implicitly assume $f_{\mathrm{BNC}}=0$ (van den Bosch et al. 2004; More et al. 2009b,a, 2011; Li et al. 2012) or that satellite galaxies cannot be brighter than $x$ times the central where $x$ is some number above unity (Klypin \& Prada 2009; Wojtak \& Mamon 2013). In studies using satellite kinematics to constrain the galaxy-halo connection, a non-zero $f_{\mathrm{BNC}}$ would lead to a systematic over-prediction of halo masses (Skibba et al. 2011). Furthermore, Tinker et al. (2017) have recently shown that determining central galaxies in galaxy groups is crucial for studying galaxy assembly bias (Zentner et al. 2014, 2016) and galactic conformity (Weinmann et al. 2006; Kauffmann et al. 2013). Finally, determining $f_{\text {BNC }}$ can inform our understanding of galaxy formation and evolution since it depends on many poorly constrained processes, such as satellite quenching, dynamical friction, and the degree to which central galaxies grow in mass by accreting satellites.

In this work, we find values for $f_{\mathrm{BNC}}\left(M, L_{\mathrm{BHG}}\right)$ that are surprisingly large compared to theoretical models and predictions from conditional luminosity functions (CLFs) (Yang et al. 2003; van den Bosch et al. 2003). Marginalized over the luminosity dependence, we find $f_{\mathrm{BNC}}(M)$ values that broadly agree with Skibba et al. (2011). We show explicitly what our best-fit values for $f_{\mathrm{BNC}}$ imply for studies of satellite kinematics, the radial profile of satellite galaxies, and galaxy-galaxy lensing. We also find that standard CLF models fail to predict the observed $f_{\mathrm{BNC}}\left(M, L_{\mathrm{BHG}}\right)$ and describe how those models could be adjusted to be in better agreement with observations. Additionally, we discuss how our results fit into galaxy formation models.

Our paper is organized as follows. In section 2 we outline how we measure the phase-space positions of BHGs with respect to nearby galaxies, primarily other halo members. In section 3 we describe how we extract the signal from the main galaxy-sample of SDSS DR7 (Abazajian et al. 2009). Next, we present how we produce detailed mock galaxy catalogues with a parametrized $f_{\mathrm{BNC}}$ and compare it to observations in section 4 . Our main results are described in section 5 and we explore the implications in section 6. Finally, our findings are summarized in section 7 . Throughout this work, we adopt a flat $\Lambda$ CDM cosmology with $\Omega_{m}=0.307$, $n=0.96$ and $\sigma_{8}=0.8228$. Relevant quantities are expressed in units of $h=H /(100 \mathrm{~km} / \mathrm{s} / \mathrm{Mpc})$.

\section{METHODS}

In this section we describe our general method to detect the fraction of haloes in which the BHG is not the central. 


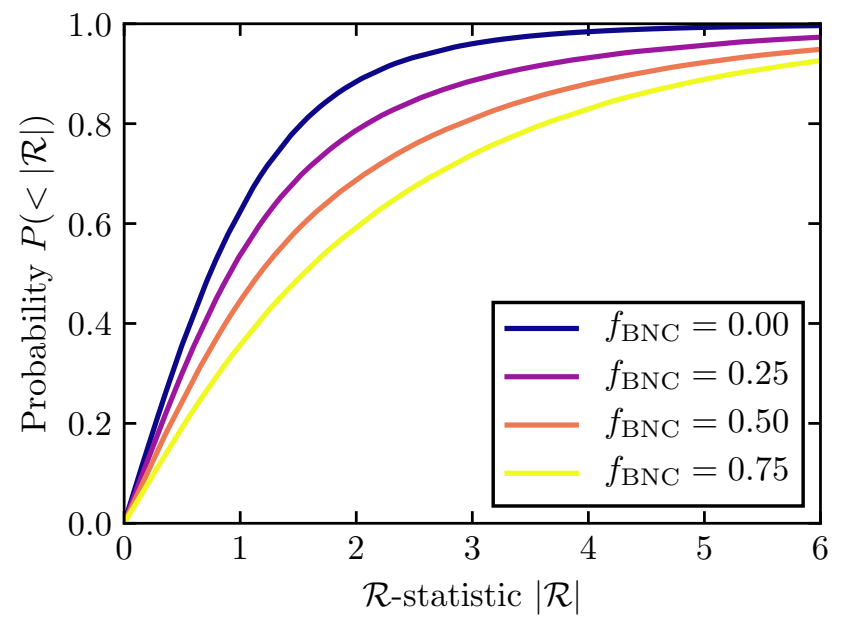

Figure 1. Demonstration of the sensitivity of the $\mathcal{R}$-statistic to the value of $f_{\mathrm{BNC}}$. We show the cumulative distribution of $|\mathcal{R}|$ for different values of $f_{\mathrm{BNC}}$, as indicated. We assume a fixed number of secondaries, $N_{\mathrm{scd}}=5$, and that satellite velocities are drawn from a Gaussian distribution while the central is at rest.

\subsection{Summary Statistics}

To analyse $f_{\mathrm{BNC}}$ we use the method introduced by van den Bosch et al. (2005b) and also used in Skibba et al. (2011). The idea is to detect spatial and velocity offsets of the BHG with respect to other halo members. In a hypothetical, fullyrelaxed halo containing an unlimited number of satellite galaxies, the average phase-space position of all galaxies in the halo should coincide with the halo centre. Thus, by comparing the position of the BHG to the galaxies in the same halo we can determine whether or not the BHG is the central galaxy. In actuality, the number of galaxies detectable in each halo is finite and halos are not fully relaxed, so the centre of an individual halo cannot be determined. Rather, we can determine $f_{\mathrm{BNC}}$ for a large ensemble of dark matter haloes.

van den Bosch et al. (2005b) introduced the $\mathcal{R}$-statistic which is designed to detect line-of-sight velocity offsets of the $\mathrm{BHG}$ with respect to neighbouring galaxies. In the following, we will refer to BHGs as primaries and their nearby galaxies as secondaries. The $\mathcal{R}$-statistic is defined for each primary as follows,

$\mathcal{R}=\sqrt{N_{\mathrm{scd}}} \frac{\left\langle v_{\mathrm{scd}}\right\rangle-v_{\mathrm{pri}}}{\hat{\sigma}_{\mathrm{scd}}}$,

where $N_{\text {scd }}$ is the number of secondaries likely associated with the same underlying halo as the primary, $\left\langle v_{\text {scd }}\right\rangle-v_{\text {pri }}$ is the average line-of-sight velocity of the secondaries with respect to the primary,

$\left\langle v_{\mathrm{scd}}\right\rangle=\frac{1}{N_{\mathrm{scd}}} \sum_{i=1}^{N_{\mathrm{scd}}} v_{i}$

and $\hat{\sigma}_{\text {scd }}$ is an estimate of the velocity dispersion for each system,

$\hat{\sigma}_{\mathrm{scd}}^{2}=\frac{1}{N_{\mathrm{scd}}-1} \sum_{i=1}^{N_{\mathrm{scd}}}\left(v_{i}-\left\langle v_{\mathrm{scd}}\right\rangle\right)^{2}$.

If all secondaries were true satellites and all $v_{i}$ were drawn from a Gaussian distribution, the $\mathcal{R}$-statistic would follow a student's t-distribution. On the other hand, if some of the brightest galaxies were satellites, the distribution would be biased towards higher values in $|\mathcal{R}|$.

Figure 1 shows the cumulative distribution of $|\mathcal{R}|$ for different values of $f_{\mathrm{BNC}}$. We take the absolute value of $\mathcal{R}$ because there is no useful information in the sign of $\mathcal{R}$. For this plot we make several idealized assumptions. For example, we assume that the number of secondaries is fixed at $N_{\mathrm{scd}}=5$ and that the line-of-sight velocities of satellites are drawn from a single Gaussian distribution. For $f_{\mathrm{BNC}}=0$, the distribution equals a student's t-distribution with virtually no values of $|\mathcal{R}|$ exceeding 6 . As we increase $f_{\mathrm{BNC}}$, higher values in $|\mathcal{R}|$ become more likely. This is the general effect we will utilize throughout this study. It is important to note that many of the assumptions going into Figure 1 are unlikely to be met in observations. For example, in general $N_{\mathrm{scd}}$ will vary from primary to primary, and the velocity dispersion of satellites likely has a radial profile $\sigma(r)$. In addition, there might be velocity anisotropy and/or non-vanishing higherorder moments of the velocity distribution (e.g., Diemand et al. 2004; Wojtak \& Mamon 2013). Thus, the line-of-sight velocities of satellite galaxies cannot be drawn from a single Gaussian distribution. Furthermore, it is not possible to identify all halo members while having no contamination from interloper galaxies. In observations some true satellite galaxies will be missed, while some other secondaries will be interloper galaxies belonging to a different halo. Finally, effects of the survey mask and spectroscopic fibre collisions can further alter the $\mathcal{R}$-distribution. Thus, it is necessary to compare observational values for the $\mathcal{R}$-distribution to detailed mock catalogues that take all of the above effects into account (van den Bosch et al. 2005b; Skibba et al. 2011).

As the $\mathcal{R}$-statistic is designed to detect line-of-sight velocity offsets, we similarly define the $\mathcal{S}$-statistic to detect spatial offsets perpendicular to the line-of-sight:

$\mathcal{S}=\sqrt{N_{\mathrm{scd}}} \frac{\sqrt{\left\langle x_{\mathrm{scd}}\right\rangle^{2}+\left\langle y_{\mathrm{scd}}\right\rangle^{2}}}{\sqrt{\hat{\sigma}_{x, \mathrm{scd}}^{2}+\hat{\sigma}_{y, \mathrm{scd}}^{2}}}$,

where $x$ and $y$ represent physical distances with respect to the primary perpendicular to the line-of-sight and the average and dispersion estimates are defined in analogy with the $\mathcal{R}$-statistic. Skibba et al. (2011) have shown that using the $\mathcal{R}$ and $\mathcal{S}$-statistic simultaneously helps to break model degeneracies. Specifically, it helps to distinguish between a scenario where $f_{\mathrm{BNC}}>0$ and a scenario in which the centrals have spatial and velocity offsets with respect to the centre of the potential well of the halo. In principle, both hypotheses affect the $\mathcal{R}$ and $\mathcal{S}$ distributions in a similar way by driving them towards more extreme values. However, if the central has spatial and velocity offsets it will have a stronger effect on the $\mathcal{R}$-statistic than on the $\mathcal{S}$-statistic because the central galaxy would need large velocity offsets before it is able to escape the centre of the potential well. Skibba et al. (2011) showed that offsets between the central galaxy and the potential well cannot simultaneously explain both the $\mathcal{R}$ and $\mathcal{S}$ distributions in SDSS data, and therefore argued for $f_{\mathrm{BNC}}>0$. 


\subsection{Isolation Criteria}

The $\mathcal{R}$ and $\mathcal{S}$ statistics require the identification of primaries and secondaries. Ideally, all BHGs should be identified as primaries while all other galaxies that reside in the same halo are identified as secondaries. van den Bosch et al. (2005b) and Skibba et al. (2011) used a group finder for this task. The brightest galaxy in each group was chosen to be the primary and all remaining galaxies in that group associated secondaries. The advantage of this method is that all galaxies in the survey can be used. However, this type of completeness comes with a number of possible failure modes. Campbell et al. (2015) identified two main group finding errors. Galaxies that are part of a single large halo may be erroneously identified as two distinct groups, a process called "fracturing," while galaxies of two distinct haloes may erroneously be associated with a single group, an error referred to as "fusing." The former case will lead to at least one primary not being a BHG and the latter one will result in secondaries that are not part of the same halo as the primary. Campbell et al. (2015) showed that around 30\% of all supposed satellites, i.e. secondaries, identified by the Yang et al. (2007) group finder are actually centrals of their own, i.e. mostly BHGs. Group identification errors are likely to influence the measured $\mathcal{R}$ and $\mathcal{S}$ distributions. If the incidence of these group errors is not reproduced in the mock catalogues used to interpret the data, group errors may lead to biases in the inferred values of $f_{\mathrm{BNC}}$.

To mitigate this potential bias we use the isolation criteria introduced in van den Bosch et al. (2004). Generally, this method uses a cylindrical isolation criteria. A galaxy is considered a primary if it is not within a cylindrical volume defined by depth $(\Delta V)_{\mathrm{h}}$ and radius $R_{\mathrm{h}}$ of another galaxy brighter by a factor $f_{\mathrm{h}}$. The cylinder is aligned with the line of sight to account for redshift space distortions. Having identified isolated galaxies as primaries, we associate secondaries to them if they lie in a smaller cylinder defined by $(\Delta V)_{\mathrm{s}}$ and radius $R_{\mathrm{s}}$ and are fainter by a factor $f_{\mathrm{s}}$. We follow van den Bosch et al. (2004) and adopt $f_{\mathrm{s}}=f_{\mathrm{h}}=1$.

Additionally, van den Bosch et al. (2004) have shown that one can achieve a very high purity and completeness in the satellites by making the cylinder sizes dependent on the luminosity of the galaxy. The idea is that the luminosity of the galaxy scales with the halo mass which in turn scales with the size of the halo. More et al. (2011) found that the average velocity dispersion as a function of the luminosity of the primary scales as

$\log \sigma_{200}=-0.11+0.37 \log L_{10}+0.30\left(\log L_{10}\right)^{2}$.

Here $\sigma_{200}$ is the satellite velocity dispersion in units of $200 \mathrm{~km} \mathrm{~s}^{-1}$, and $L_{10}=L /\left(10^{10} h^{-2} \mathrm{~L}_{\odot}\right)$.

For this work we adopt $(\Delta V)_{\mathrm{s}}=(\Delta V)_{\mathrm{h}}=5 \sigma=$ $1000 \sigma_{200} \mathrm{~km} \mathrm{~s}^{-1}, R_{\mathrm{s}}=0.25 \sigma_{200} h^{-1} \mathrm{Mpc}$ and $R_{\mathrm{h}}=3.2 R_{\mathrm{s}}$. Since Eq. (5) implies unrealistically high values for the satellite velocity dispersion for a few extremely bright galaxies, specifically those without spectroscopic redshift, we cap the satellite velocity dispersion to $1000 \mathrm{~km} \mathrm{~s}^{-1}$ when specifying our cylinders. These values are chosen as a compromise between completeness and contamination. Altogether, we can achieve interloper fractions of around $18 \%$, as measured in our mock catalogues described in section 4 . In other words, $82 \%$ of all secondaries are galaxies living in the same halo as the primary. Similarly, in a mock with $f_{\mathrm{BNC}}=0$, we find that more than $99 \%$ of all primaries are true BHGs.

We note that in this method, some galaxies are classified as neither primaries nor secondaries. In principle, this does not pose a problem because the $\mathcal{R}$ and $\mathcal{S}$-statistics do not require all galaxies in the same halo to be identified. However, we point out an asymmetry in satellite identification: completeness in true satellites is typically close to unity in the line-of-sight direction around primaries, but incompleteness is larger in the transverse direction because galaxies beyond a certain projected distance are not included in the calculation. This incompleteness reduces the sensitivity of the $\mathcal{S}$-statistic but does not bias our estimator.

\section{OBSERVATIONS}

In this work, we use data from the SDSS DR7 main galaxy sample (Padmanabhan et al. 2008; Abazajian et al. 2009). In particular, we work with the bright0 sample of the New York University Value-Added Galaxy Catalog (NYU-VAGC; Blanton et al. 2005). This catalogue includes all galaxies of the SDSS main galaxy sample with an apparent magnitude $m_{r}<17.6$. Due to the design of the SDSS survey, if two galaxies are separated by less than $55^{\prime \prime}$, spectroscopic fibres cannot be placed for both of them. Due to these fibre collisions a small fraction of the galaxies lack spectroscopic redshifts. In the bright0 sample, galaxies lacking a spectroscopic redshift have been assigned the redshift of the closest neighbour. We convert apparent $r$-band magnitudes into luminosities k-corrected (Blanton \& Roweis 2007) and evolution corrected to $z=0.1$. Of the bright0 sample we select those galaxies with $0.01 \leqslant z \leqslant 0.17$ and an r-band luminosity $L>10^{9} h^{-2} \mathrm{~L}_{\odot}$. Roughly 500,000 of the 570,000 galaxies in the original sample pass this cut. The redshift and luminosity limits are determined primarily by the volume and resolution of our mock catalogues, as discussed in the next section.

As the next step we run the isolation criteria discussed in the previous section on this sample. After having identified primaries and their associated secondaries we apply additional selection cuts. We require that all primaries have a spectroscopic redshift, i.e. no fibre collision, and lie in a region of SDSS DR7 with at least $80 \%$ redshift completeness. We stress that we apply the isolation criteria using all galaxies, i.e. also those with fibre collisions. In that case we use the redshift of the closest neighbour as given in the bright0 sample. The reason is that the true BHG might itself not have a spectroscopic redshift. Excluding this galaxy before applying the isolation criteria would result in the second brightest galaxy of the halo being wrongly identified as a primary. Next, we require the primary to have a redshift in the range $0.03 \leqslant z \leqslant 0.15$. This redshift cut is more restrictive than the previous one and designed to avoid edge effects in redshift space. This more restrictive cut is not applied to associated secondaries around that primary. Particularly, the redshift range for secondaries is larger by 0.02 , corresponding to $\sim 6000 \mathrm{~km} \mathrm{~s}^{-1}$, which is larger than the maximum $(\Delta V)_{\mathrm{s}}$ of $5000 \mathrm{~km} \mathrm{~s}^{-1}$. Furthermore, we exclude galaxies that lie close to the survey edge. We quantify this by calculating an angular completeness score for every galaxy. The completeness score is defined as the fraction of the area 


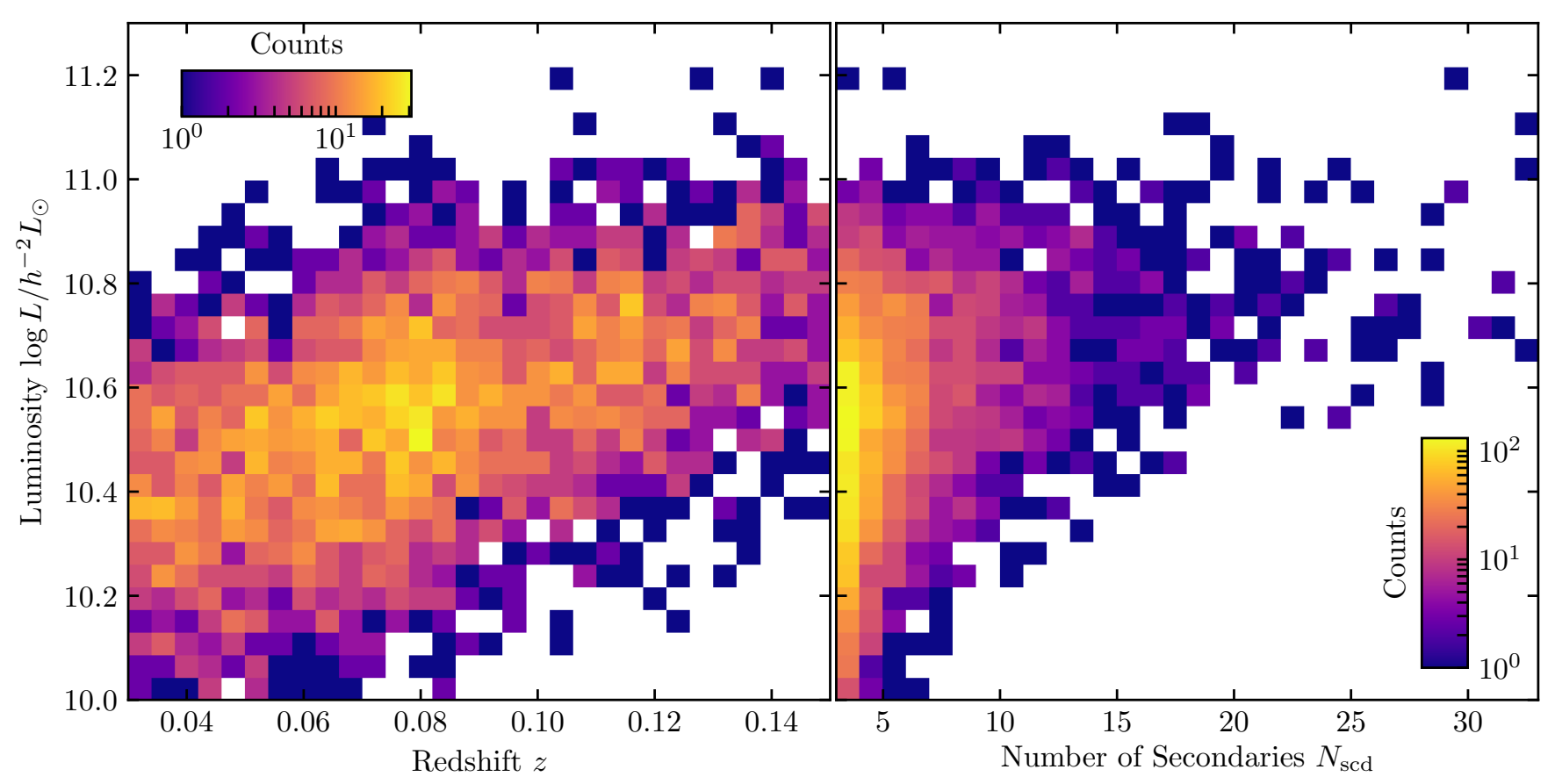

Figure 2. Properties of primaries in SDSS DR7. The left-hand panel shows a histogram of the luminosities of the primaries against their redshifts. Due to the flux limit of SDSS, there is a positive correlation of redshift with luminosity. The right-hand panel is similar with the $\mathrm{x}$-axis being the number of associated secondaries. Most primaries have less than 10 secondaries.

defined by the cylinder given by $R_{\mathrm{h}}$ around each galaxy that lies within the window function of the bright0 sample. We require a completeness score of at least $90 \%$ for primaries. Again, this cut is not applied to associated secondaries. We demand that primaries have $L \geqslant 10^{10} h^{-2} \mathrm{~L}_{\odot}$ and at least 3 secondaries with spectroscopic redshifts. Finally, following Skibba et al. (2011), we require $\hat{\sigma}_{\text {scd }} \geqslant 50 \mathrm{~km} \mathrm{~s}^{-1}$.

Upon applying these selections, we arrive at a sample of roughly 3600 primaries and calculate the $\mathcal{R}$ and $\mathcal{S}$-statistics for each primary using only secondaries with spectroscopic redshifts. We show the luminosity and redshift distributions of the primaries in Figure 2. As seen in the left-hand panel, there is a general trend of increasing luminosity with redshift that is due to the flux limit of SDSS DR7. In the same Figure we also show the distribution of the number of secondaries around each primary. We find that most primaries have 3 to 10 secondaries. Also, there are more secondaries around brighter primaries. Altogether, we find around 20, 000 secondaries in our sample.

\section{MOCK CATALOGUES}

In this section we describe how we create mock catalogues for parameter inference. Generally, we create mock galaxy catalogues similar to the bright0 sample of the NYU-VAGC and then process them using the same pipeline as for the real data.

\subsection{Dark Matter Simulation}

In this work we use results from the Small MultiDark Planck (SMDPL; Klypin et al. 2016) dark-matter-only $N$ - body simulation. The simulation traces the evolution of dark matter structures in a cubic volume of $400 h^{-1} \mathrm{Mpc}$ on a side using $3840^{3}$ particles, resulting in a particle mass of $9.6 \times 10^{7} h^{-1} \mathrm{M}_{\odot}$. The cosmological parameters of this simulation are compatible with results from the Planck satellite (Planck Collaboration et al. 2014). The simulation output has been analysed with the ROCKSTAR halo finder (Behroozi et al. 2013) from which we use the redshift $z=0.1$ halo catalogue ${ }^{1}$. This redshift roughly corresponds to the median redshift of primaries in our SDSS sample. Furthermore, we require dark matter (sub)haloes in the halo catalogue to have $M_{\text {peak }}$, the maximum halo mass achieved over the lifetime of the (sub)halo, exceeding $\sim 3 \times 10^{10} h^{-1} \mathrm{M}_{\odot}$, which is 300 times the dark matter particle mass. This ensures that all (sub)haloes in the catalogue are well resolved.

\subsection{Galaxy Occupation}

We use halotools (Hearin et al. 2016) to populate dark matter haloes from the SMDPL simulation output with galaxies. In particular, we use a CLF (Yang et al. 2003) approach, the parametrization of Cacciato et al. (2009) and the bestfit Fiducial parameters from Cacciato et al. (2013). These parameters are listed in Table 1.

The occupation of dark matter haloes with galaxies is split into a central and satellite galaxy part,

$\Phi(L \mid M)=\Phi_{\mathrm{c}}(L \mid M)+\Phi_{\mathrm{s}}(L \mid M)$,

where $L$ is the luminosity of the galaxy (in units of $h^{-2} \mathrm{~L}_{\odot}$ ) and $M$ the mass of the dark matter halo (in units of $h^{-1} \mathrm{M}_{\odot}$ ).

1 http://yun.ucsc.edu/sims/SMDPL/hlists/index.html 


\begin{tabular}{cc} 
Parameter & Value \\
\hline \hline $\log L_{0} /\left(h^{-2} \mathrm{~L}_{\odot}\right)$ & 9.95 \\
$\log M_{1} /\left(h^{-1} \mathrm{M}_{\odot}\right)$ & 11.24 \\
$\sigma_{\log L}$ & 0.157 \\
$\gamma_{1}$ & 3.18 \\
$\gamma_{2}$ & 0.245 \\
$\alpha_{\mathrm{S}}$ & -1.18 \\
$b_{0}$ & -1.17 \\
$b_{1}$ & 1.53 \\
$b_{2}$ & -0.217 \\
\hline
\end{tabular}

Table 1. Parameters of the default CLF parametrization used in this work.

Following Cacciato et al. (2013), the dark matter halo mass is taken to be the mass defined with respect to 200 times the background density of the Universe, $M_{200 b}$. The median luminosity of central galaxies is parametrized by

$\left\langle\log L_{\mathrm{c}}(M)\right\rangle=\log \left(L_{0} \frac{\left(M / M_{1}\right)^{\gamma_{1}}}{\left[1+\left(M / M_{1}\right)\right]^{\gamma_{1}-\gamma_{2}}}\right)$,

where $L_{0}, M_{1}, \gamma_{1}$ and $\gamma_{2}$ are parameters taken from Cacciato et al. (2013), see Table 1. Each halo from the halo catalogue is assigned a central galaxy and luminosities are drawn from a log-normal distribution with median $\log L_{\mathrm{c}}(M)$ and scatter $\sigma_{\log L}=0.157$, i.e.

$\Phi_{\mathrm{c}}(L \mid M)=\frac{\log e}{\sqrt{2 \pi \sigma_{\log L}^{2}} L} \exp \left[-\frac{\left(\log L-\left\langle\log L_{\mathrm{c}}(M)\right\rangle\right)^{2}}{2 \sigma_{\log L}^{2}}\right]$.

The satellite CLF is parametrized by

$\Phi_{\mathrm{s}}(L \mid M)=\frac{\Phi_{\mathrm{s}}^{\star}(M)}{L_{\star}}\left(\frac{L}{L_{\star}}\right)^{\alpha_{\mathrm{s}}} \exp \left[-\left(\frac{L}{L_{\star}}\right)^{2}\right]$

with

$\log \Phi_{\mathrm{s}}^{\star}(M)=b_{0}+b_{1} \log M_{12}+b_{2}\left(\log M_{12}\right)^{2}$

and

$\log L_{\star}(M)=\left\langle\log L_{\mathrm{c}}(M)\right\rangle-0.25$,

which describes the exponential cut-off of the satellite luminosity function. Here $M_{12}=M /\left(10^{12} h^{-1} \mathrm{M}_{\odot}\right)$ and $\alpha_{\mathrm{s}}, b_{0}, b_{1}$ and $b_{2}$ are parameters (see Table 1 ) again taken from Cacciato et al. (2013). The number of satellites is determined by first integrating Eq. (9) over the relevant luminosity range. This gives the expected number of satellites. We then assume that the actual number follows a Poisson distribution. Note that for the moment we assume the faint-end slope $\alpha_{\mathrm{s}}$ of the satellite luminosity function to be independent of the halo mass. Also, it is noteworthy that the best-fit parameters of Cacciato et al. (2013) have been derived for different cosmological parameters and partially using data-sets different from SDSS DR7. We will explore the robustness of our results to reasonable changes in the above choice of parameters in section 5.2 .

Finally, we stress that for the moment we allow satellites to be brighter than their respective centrals. In fact, for a given CLF parametrization, the fraction of haloes of mass $M$ and with a brightest halo galaxy of luminosity $L_{\mathrm{BHG}}$ in which this brightest galaxy is not the central, which we denote by $f_{\mathrm{BNC}}\left(M, L_{\mathrm{BHG}}\right)$, is fully determined. It can be expressed via

$$
f_{\mathrm{BNC}}\left(L_{\mathrm{BHG}}, M\right)=\frac{\Phi_{\mathrm{s}}\left(L_{\mathrm{BHG}} \mid M\right)}{\frac{\Phi_{\mathrm{c}}\left(L_{\mathrm{BHG}} \mid M\right)}{1-\int_{L_{\mathrm{BHG}}}^{\infty} \Phi_{\mathrm{c}}(L \mid M) \mathrm{d} L}+\Phi_{\mathrm{s}}\left(L_{\mathrm{BHG}} \mid M\right)} .
$$

The first term in the denominator accounts for the possibility that the brightest galaxy is the central, while the second term and the term in the numerator represent the case of the satellite being the brightest galaxy. Note that the first term is not just $\Phi_{\mathrm{c}}\left(L_{\mathrm{BHG}} \mid M\right)$, but boosted by $\left[1-\int_{L_{\mathrm{BHG}}}^{\infty} \Phi_{\mathrm{c}}(L \mid M) \mathrm{d} L\right]^{-1}$. The reason is that we assume that all haloes have exactly one central which increases $\Phi_{\mathrm{C}}$ once we know that no central is brighter than $L_{\mathrm{BHG}}$. This and other useful relations for CLF models are described in the appendix. We will later swap phase-space positions of centrals and satellites to re-parametrize $f_{\mathrm{BNC}}$, as described in section 4.4. Finally, note that $f_{\mathrm{BNC}}$ as predicted by the CLF model depends sensitively on the exponential cut-off of the satellite luminosity function given in Eq. (9) and (11) (Skibba et al. 2011). In section 6.3, we will test the impact of variations to the above parametrization on the prediction for $f_{\mathrm{BNC}}$.

\subsection{Phase-Space Coordinates}

\subsubsection{Central galaxy velocities}

The phase-space coordinates of central galaxies are derived from the properties of their host haloes. It has been shown that central galaxies are unlikely to be at rest with respect to the bulk velocity of the entire halo. For example, Guo et al. (2015a,b), analysing the redshift-space clustering of galaxies in the SDSS DR7 and BOSS survey, have argued that central galaxies must have additional velocity offsets of the order of $25 \%$ of the dark matter particle velocity dispersion of the host halo. Additionally, the authors have shown that the strength of these velocity offsets increases with halo mass. Similar conclusions were reached by Lauer et al. (2014). Intriguingly, this kind of velocity offset is similar to that of halo cores with respect to the bulk velocity seen in dark matter simulations (Behroozi et al. 2013). Indeed, further studies have shown that central galaxies trace the dark matter halo core much better than the centre-ofmass of the entire halo (Guo et al. 2016; Ye et al. 2017). Because van den Bosch et al. (2005b) have shown that such velocity offsets of the central from the overall halo can have considerable impact on the $\mathcal{R}$-statistic, it is important to take this effect into account when modelling $f_{\mathrm{BNC}}$. Deriving $f_{\mathrm{BNC}}$ without it, as done in Skibba et al. (2011), could lead to an overestimation of $f_{\mathrm{BNC}}$. In this work, the position of the central coincides with the dark matter density peak of the host halo and the velocity is set to the dark matter core velocity. The latter is defined as the center-of-mass velocity of the particles that enclose the inner 10 percent of the host halo radius (Behroozi et al. 2013).

\subsubsection{Satellite galaxy velocities}

Satellite galaxy phase-space positions are assigned based on the phase-space positions of actual subhaloes inside $R_{\mathrm{vir}}$. For 


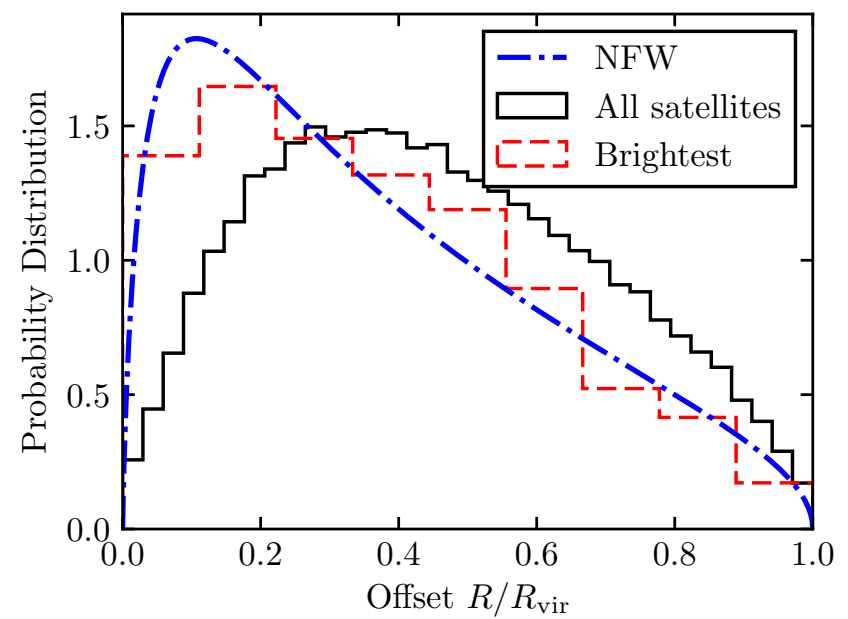

Figure 3. Comparison of the spatial distributions of all satellite galaxies (black, solid) against the brightest satellites (red, dashed) and the dark matter distribution (blue, dot-dashed) in our mock galaxy catalogues. In all haloes, the phase-space position of the brightest satellite is that of the subhalo with the highest $M_{\text {peak }}$. The dark matter distribution is modelled with an NFW profile where the concentration parameter equals the median of all host halos. Our model implies that satellite galaxies are anti-biased with respect to the dark matter and that the brightest satellite galaxies are more centrally concentrated than the entire satellite galaxy population.

every halo in the simulation we first draw satellite galaxy numbers and luminosities from the CLF assuming a Poisson distribution. We then rank-order the satellite galaxies in each halo according to their luminosity. Next, we assign phase-space positions of subhaloes in the same halo rankordered by their $M_{\text {peak }}$ value. Note that the number of satellites is drawn independently of the number of resolved subhaloes that are present inside a host halo. Consequently, it is possible that the number of satellite galaxies assigned to a halo exceeds the number of subhaloes. If that occurs, we take relative phase-space positions from random subhaloes hosted by haloes of a similar mass and apply a random angular orientation. However, even for the lowest luminosity threshold used in this analysis, this only needs to be done for $\sim 5 \%$ of all the satellite galaxies.

This novel approach combines the flexibility of the traditional CLF method with the predictive power of subhalo abundance matching (SHAM) (e.g., Vale \& Ostriker 2004; Conroy et al. 2006; Guo et al. 2010; Hearin et al. 2013b). Because subhaloes are spatially anti-biased with respect to the dark matter distribution (Diemand et al. 2004), our model implies the same for satellite galaxies. Additionally, since we assume a direct correlation between $M_{\text {peak }}$ and $L$, any segregation with respect to $M_{\text {peak }}$ (van den Bosch et al. 2016) manifests itself as a luminosity segregation. In particular our approach implies that the brightest satellite galaxies are radially more concentrated than the overall satellite galaxy population. This is demonstrated in Figure 3 where we show the projected core separations for satellite galaxies above a luminosity threshold of $10^{9} \mathrm{~L}_{\odot}$ in haloes of $10^{14} h^{-1} \mathrm{M}_{\odot} \leqslant \mathrm{M}_{\text {vir }} \leqslant 3 \times 10^{14} \mathrm{~h}^{-1} \mathrm{M}_{\odot}$ as the solid, black histogram. This should be compared to both the distribution of core separations of the brightest satellite galaxy (red, dashed histogram) and the dark matter distribution described by a Navarro-Frenk-White (NFW, Navarro et al. 1996) profile (blue, dash-dot line) with a concentration parameter set to 5.4 , the median concentration of all host halos. These results are in good qualitative agreement with the observational results of Hoshino et al. (2015).

\subsubsection{Correlated satellite velocities}

As mentioned in Skibba et al. (2011), satellite velocities might be correlated among each other. For example, two recently merged haloes might be severely unrelaxed, resulting in strong phase-space correlations of the satellite population. Also, the filamentary structure of the cosmic web might introduce similar effects. Skibba et al. (2011) have shown that ignoring such correlations can bias the inferred value of $f_{\mathrm{BNC}}$ high. Because they did not take this effect into account, they regarded their derived values for $f_{\mathrm{BNC}}$ as upper limits. Our approach for assigning phase-space coordinates of satellites naturally accounts for such correlations.

Let us first explore the presence of satellite velocity correlations. To quantify this effect, we compute

$\mathcal{C}=\sqrt{\left\langle\frac{\left\langle\left(v_{i}-v_{\mathrm{c}}\right)\left(v_{j}-v_{\mathrm{c}}\right)\right\rangle_{\mathrm{sub}}}{V_{\text {vir }}^{2}}\right\rangle}$,

where $v_{i}$ and $v_{j}$ are the $z$-velocities of distinct subhaloes, $v_{\mathrm{c}}$ is the velocity of the halo core, \langle\rangle denotes the average over all haloes and \langle\rangle$_{\text {sub }}$ the average over all subhaloes of a particular halo. Ideally, because satellite galaxies sample the entire halo, and not merely the core region, we would want to express subhalo velocities relative to the bulk velocity of the halo, rather than its core velocity. However, these phasespace positions are not available in the publicly available halo catalogues. As a consequence, we already expect $\mathcal{C}>0$, simply due to the velocity offsets of halo cores. In order to account for this, we proceed as follows. Let us assume that there are no correlations between subhalo and core velocities in the rest-frame of the entire halo, so that $\left\langle v_{i} v_{j}\right\rangle_{\mathrm{sub}}=0$ for $i \neq j$ and $\left\langle v_{i} v_{\mathrm{c}}\right\rangle_{\mathrm{sub}}=0$. Then we find

$\mathcal{C}=\sqrt{\left\langle\frac{v_{\mathrm{c}}^{2}}{V_{\text {vir }}^{2}}\right\rangle}$.

Hence, if the above assumption of no satellite correlation is true, $\mathcal{C}$ will be the same, but not necessarily zero, regardless of the subhalo population used. We can assess this by splitting the subhaloes in 'young' and 'old' subpopulations according to $M_{\text {vir }}>M_{\text {peak }} / 3$ and $M_{\text {vir }} \leqslant$ $M_{\text {peak }} / 3$, respectively ${ }^{2}$. We compute $\mathcal{C}$ over all haloes with $13 \leqslant \log M_{\text {vir }} /\left(h^{-1} \mathrm{M}_{\odot}\right) \leqslant 14$ that have at least 3 young and 3 old subhaloes. Unbound subhaloes and higher-order subhaloes (i.e., sub-subhaloes, etc.) have been excluded for this analysis. We find $\mathcal{C}=0.171 \pm 0.003$ and $\mathcal{C}=0.090 \pm 0.004$ for young and old subhaloes, respectively. On the other hand, using all subhaloes we find $\mathcal{C}=0.122 \pm 0.004$. Again, if the

2 Here the notion is that old subhaloes were accreted earlier, and have therefore experienced more mass loss (van den Bosch et al. 2005a; Zentner et al. 2005). 
non-vanishing $\mathcal{C}$ would be entirely due to core velocity offsets, those values should be identical. Instead, the data suggests that there are additional correlations in the velocities of recently accreted subhaloes, i.e. $\left\langle v_{i} v_{j}\right\rangle_{\mathrm{sub}}>0$.

\subsubsection{Summary}

To summarize, we assign phase-space positions for central and satellite galaxies using the phase-space positions of halo centres and subhaloes in the simulation from which the dark matter halo catalogs are generated. In this way, we account for the unrelaxed state of the haloes, including halo core velocity offsets, subhalo velocity correlations, and asphericity. Most of these effects increase the absolute values of the $\mathcal{R}$ and $\mathcal{S}$-statistics. Thus, taking it into account is important to get an unbiased estimate of $f_{\mathrm{BNC}}$.

\subsection{Parametrizing $f_{\mathrm{BNC}}$}

So far, our choice of the CLF model implicitly determines $f_{\mathrm{BNC}}$ as a function of halo mass and luminosity of the BHG (Eq. [12]). However, as shown in section 6.3, the best-fit $f_{\mathrm{BNC}}$ implied by the data is considerably different than the CLF prediction. Since the main goal of this work is to model $f_{\mathrm{BNC}}$ regardless of the detailed CLF choice, we re-parametrize $f_{\mathrm{BNC}}$. First, for each halo in the catalogue we swap the luminosities of the central and the brightest satellite galaxy if the brightest satellite is brighter than the central. This implies $f_{\mathrm{BNC}}=0$ for all haloes. This procedure is similar to the one in Skibba et al. (2011). At this point, the question is how to model the possibility that some satellite galaxies are brighter than the central. Skibba et al. (2011) assumed that this probability is dependent only on the mass of the dark matter halo. Generally, this probability should also depend on the luminosity of the BHG. This did not affect the results of Skibba et al. (2011) since the BHG luminosity was not used in their analysis. However, because we explicitly use $L_{\mathrm{BHG}}$ as an observable, we need to parametrize $f_{\mathrm{BNC}}$ as a function of both halo mass and BHG luminosity.

Furthermore, there is a third, less intuitive dependence that is important to consider. In principle, the probability of a satellite being brighter than the central should have a correlation with the satellite occupation. If a halo has more satellites, it should be more likely that one of those satellites turns out to be brighter than the central. For example, CLF models commonly assume that satellite luminosities have no correlations among each other. Furthermore, it is assumed that the actual number of satellites $n_{\text {sat }}$ is related to the expected number of satellites $\left\langle n_{\text {sat }}\right\rangle$ above the detection threshold $L_{\mathrm{th}}$,

$\left\langle n_{\mathrm{sat}}\right\rangle(M)=\int_{L_{\mathrm{th}}}^{\infty} \Phi_{\mathrm{s}}(L \mid M) d L$,

via a Poisson distribution. As shown in the appendix, the number $n_{\mathrm{BTC}}$ of satellites brighter than the central of luminosity $L_{\mathrm{c}}$ also follows a Poisson distribution with an expected value

$\left\langle n_{\mathrm{BTC}}\right\rangle\left(L_{\mathrm{c}}, M\right)=\int_{L_{\mathrm{c}}}^{\infty} \Phi_{\mathrm{s}}(L \mid M) d L$.
Most importantly the number of satellites fainter than $L_{\mathrm{c}}$, for which the expectation value is $\left\langle n_{\mathrm{FTC}}\right\rangle=$ $\int_{L_{\mathrm{th}}}^{L \mathrm{c}} \Phi_{\mathrm{S}}(L \mid M) d L$, is independent of $n_{\mathrm{BTC}}$. Hence, we have that $\left\langle n_{\mathrm{sat}}\right\rangle=\left\langle n_{\mathrm{BTC}}\right\rangle+\left\langle n_{\mathrm{FTC}}\right\rangle$. If $\left\langle n_{\mathrm{BTC}}\right\rangle$ is small such that $n_{\mathrm{BTC}}=0$ or 1 , we thus see that $n_{\text {sat }}$ will on average be higher by 1 for systems where the brightest galaxy is not the central $\left(n_{\mathrm{BTC}}=1\right)$. In other words, the common assumptions of CLF modelling imply a positive correlation between the probability $p_{\mathrm{BNC}}$ that the $\mathrm{BHG}$ is the satellite and the satellite occupation $n_{\text {sat }}$. Taking this correlation between $n_{\text {sat }}$ and $p_{\mathrm{BNC}}$ into account is important because we applied a cut on the number of secondaries, i.e. satellites, when constructing our sample. Given how strongly the number of primaries decreases for a given cut in the number of secondaries, as shown in Figure 2, our selection is biased towards systems where $n_{\text {sat }}$ is larger than its expectation value.

While a correlation between satellite occupation and $p_{\text {BNC }}$ is implied by our assumed CLF, it does not need to be true for the actual Universe. We investigate the realism of such a correlation by analysing the galaxy catalogue of the semi-analytic model of De Lucia \& Blaizot (2007) which was run on the dark matter halo catalogue of the Millennium simulation (Springel et al. 2005). The motivation behind using a semi-analytic model is to explore statistics in a rich mock galaxy sample for which the correct answer is known. We first bin haloes in the halo catalogue by their host halo mass. For each small bin in host halo mass we determine a luminosity threshold such that the average satellite occupation in that halo mass bin is 3 . We apply this cut primarily because most primaries in our sample have roughly 3 secondaries. We now have a sample of haloes in which the average number of satellites $\left\langle n_{\text {sat }}\right\rangle$ is 3 , regardless of halo mass. In the left panel of Figure 4 we show $\left\langle n_{\text {sat }}\right\rangle_{\mathrm{BNC}}-\left\langle n_{\text {sat }}\right\rangle$, the difference in satellite occupation between haloes in which the central is not the BHG and the general population. If $p_{\mathrm{BNC}}$ were uncorrelated with $n_{\text {sat }}$, this difference should be 0 . On the other hand, assuming that satellite luminosities are uncorrelated and that $\left\langle n_{\mathrm{BTC}}\right\rangle \ll 1$ would imply a difference of $\sim 1$. Indeed, we do find $\left\langle n_{\text {sat }}\right\rangle_{\mathrm{BNC}}-\left\langle n_{\text {sat }}\right\rangle \approx 1$ for all halo masses.

Given that central galaxies can grow by the accretion of satellites, we expect a negative correlation between central luminosity $L_{\mathrm{c}}$ and satellite occupation $n_{\text {sat }}$. To show this we divide the De Lucia \& Blaizot (2007) galaxy catalogue at each halo mass into quartiles of $L_{\mathrm{c}}$. The right-hand panel of Figure 4 displays the satellite occupation difference between central galaxies in the specific quartile compared to all centrals. We find that the most luminous central galaxies $(75 \%-100 \%)$ have on average fewer satellites. Conversely, haloes occupied by the least luminous centrals $(0 \%-25 \%)$ have a higher satellite occupation on average. Furthermore, we do expect that $p_{\mathrm{BNC}}$ has a negative correlation with $L_{\mathrm{c}}$, in that it is less likely for satellites to be brighter than the central if the latter is more luminous. These anti-correlations between $L_{\mathrm{c}}$ and $n_{\text {sat }}$ and between $L_{\mathrm{c}}$ and $p_{\mathrm{BNC}}$ also imply a positive correlation between $n_{\text {sat }}$ and $p_{\text {BNC }}$. However, we have verified that the qualitative trend in the left-hand panel of Figure 4 is not caused by this correlation with $L_{\mathrm{c}}$. Instead, even when first selecting centrals in a narrow range of $L_{\mathrm{c}}$, the difference of order unity remains. We conclude that the 

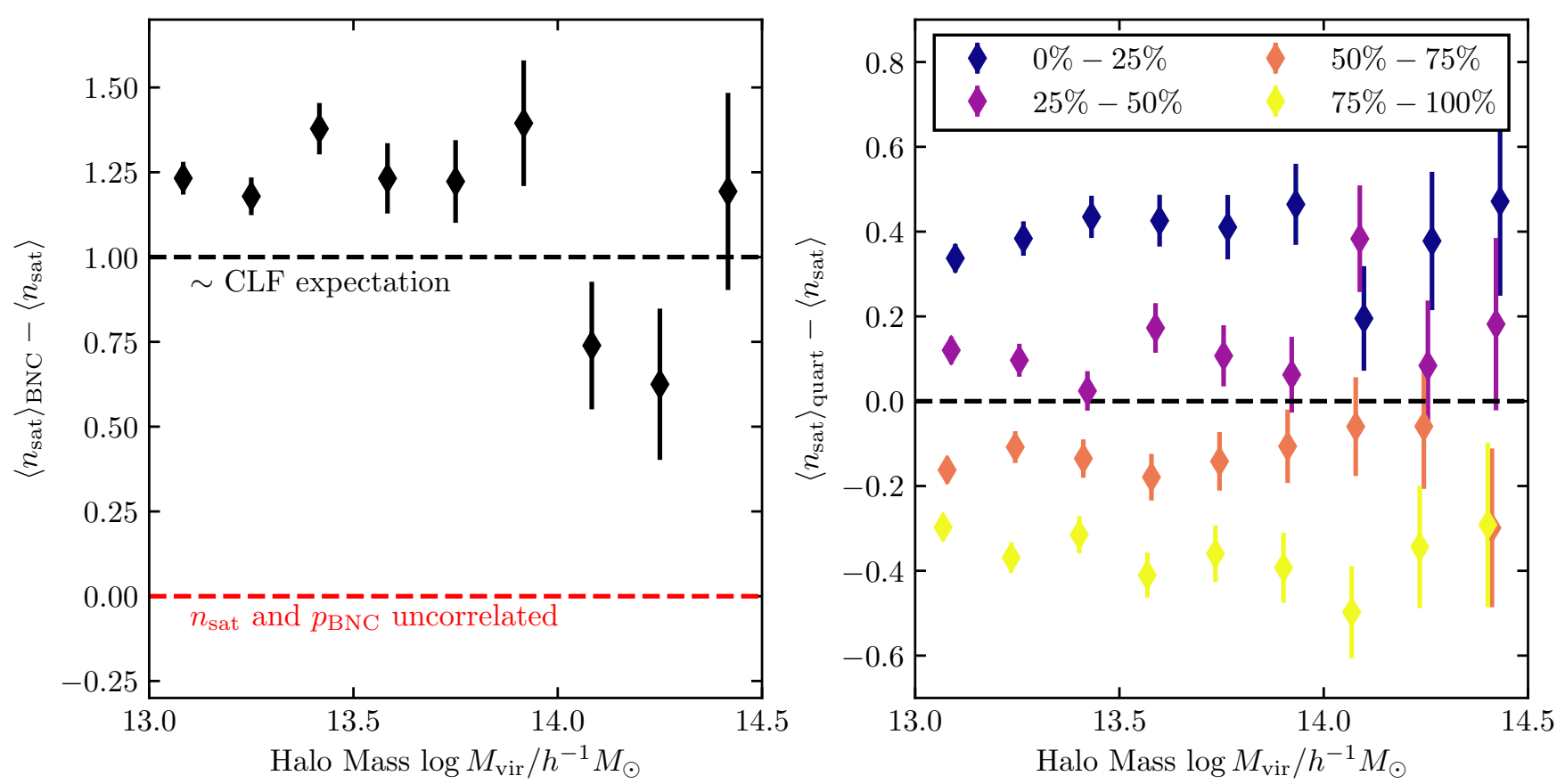

Figure 4. Results from the semi-analytic model of galaxy formation (De Lucia \& Blaizot 2007) run on the Millennium simulation. The left-hand panel shows the difference in satellite occupation between haloes where the brightest galaxy is a satellite and all haloes. Generally, the difference is roughly unity, following the CLF expectation if satellite luminosities are uncorrelated and the satellite number follows a Poisson distribution. The right-hand panel presents a similar occupation difference but now for haloes binned in quartiles of their central luminosity. Note that, at fixed halo mass, haloes with a brighter central have fewer satellites.

positive correlation between $p_{\mathrm{BNC}}$ and $n_{\text {sat }}$ implied by CLF models seems to be a good approximation even for realistic galaxy formation models.

With the above discussion in mind, what is the relation between $n_{\text {sat }}$ and $p_{\mathrm{BNC}}$ in CLF models? Once the number of satellites is specified, the probability that at least one of them is brighter than the central is given by

$p_{\mathrm{BNC}}\left(n_{\mathrm{sat}} \mid\left\langle n_{\mathrm{sat}}\right\rangle,\left\langle n_{\mathrm{BTC}}\right\rangle\right)=1-\left(1-\frac{\left\langle n_{\mathrm{BTC}}\right\rangle}{\left\langle n_{\mathrm{sat}}\right\rangle}\right)^{n_{\mathrm{sat}}}$.

On the other hand, averaged over all realizations of $n_{\text {sat }}$ one finds

$$
\begin{aligned}
f_{\mathrm{BNC}}\left(L_{c}, M\right) & =\sum_{n_{\mathrm{sat}}} p_{\mathrm{BNC}}\left(n_{\mathrm{sat}} \mid\left\langle n_{\mathrm{sat}}\right\rangle,\left\langle n_{\mathrm{BTC}}\right\rangle\right) p\left(n_{\mathrm{sat}} \mid\left\langle n_{\mathrm{sat}}\right\rangle\right) \\
& =1-\exp \left[-\left\langle n_{\mathrm{BTC}}\right\rangle\left(L_{c}, M\right)\right]
\end{aligned}
$$

Thus, to model $f_{\mathrm{BNC}}$ we need a parametrization for $\left\langle n_{\mathrm{BTC}}\right\rangle$. Note that the above equation gives the probably that, in a halo of mass $M$, the BHG is not the central, given a central luminosity $L_{\mathrm{c}}$, whereas Eq. (12) gives the same probability, given a $\mathrm{BHG}$ of luminosity $L_{\mathrm{BHG}}$. Note that, whereas the latter is an observable quantity, the former is not, as it requires perfect a priori knowledge of which galaxy is the actual central. Thus, we choose to parametrize $\left\langle n_{\mathrm{BTC}}\right\rangle$ as a function of $L_{\mathrm{BHG}}$. Under the assumption that the halo mass and BHG luminosity dependencies are independent, we therefore adopt the following fitting function for $\left\langle n_{\mathrm{BTC}}\right\rangle$,

$$
\begin{aligned}
& \left\langle n_{\mathrm{BTC}}\right\rangle\left(L_{\mathrm{BHG}}, M\right)= \\
& f_{0}+a_{1} \log L_{10}+a_{2}\left(\log L_{10}\right)^{2}+b \log M_{12},
\end{aligned}
$$

where $f_{0}, a_{1}, a_{2}$ and $b$ are free parameters, $M_{12}$ is the halo mass $M_{200 b}$ in units of $10^{12} h^{-1} \mathrm{M}_{\odot}$, and $L_{10}$ is the BHG luminosity in units of $10^{10} h^{-2} \mathrm{~L}_{\odot}$. The above parametrization is motivated by the $f_{\mathrm{BNC}}\left(L_{\mathrm{BHG}}, M\right)$ dependence for CLF models, as described in more detail in section 6.3. Note that due to our parametrization it is possible that $\left\langle n_{\mathrm{BTC}}\right\rangle>\left\langle n_{\text {sat }}\right\rangle$. In this case we set $p_{\mathrm{BNC}}$ to unity. When constructing mock catalogues, we compute $p_{\mathrm{BNC}}$ for each system via Eq. (17), taking into account both the expected and actual satellite occupation. We then draw a random number between 0 and 1 and if that number is below $p_{\mathrm{BNC}}$, we swap the phase-space coordinates of the central, which was the BHG so far, and the brightest satellite. Note that although $n_{\mathrm{BTC}}$ can be larger than unity, this method of swapping central and satellite never sets $n_{\mathrm{BTC}}$ larger than unity, i.e. the central is at least the second brightest galaxy. However, this is not crucial because for our observations we are only concerned with the phase-space position of the brightest galaxy and ignore the luminosities of secondaries. Finally, note that swapping phase-space positions in the above described way does conserve the total CLF, $\Phi_{\mathrm{c}}+\Phi_{\mathrm{s}}$, and BHG luminosity distribution $\Phi_{\mathrm{BHG}}$ for each halo, while modifying the central and satellite CLF.

\subsection{Mock SDSS Surveys}

To create mock SDSS-like galaxy surveys we first populate all dark matter haloes in the SMDPL simulation assuming a constant luminosity threshold of $10^{9} h^{-2} \mathrm{~L}_{\odot}$. We then place a virtual observer with random orientation at a random position inside the simulation box. By periodically repeating the galaxy catalogue if needed we calculate all the galaxies 


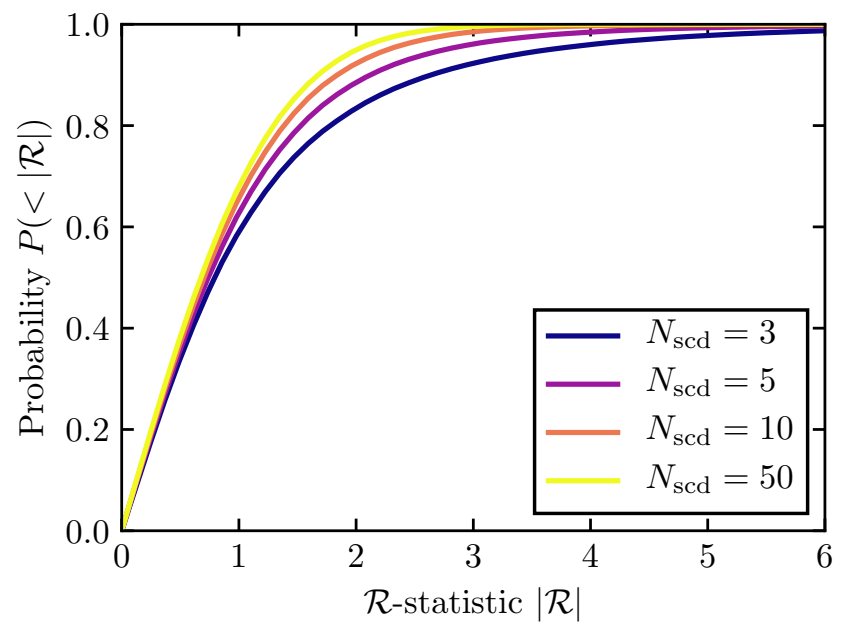

Figure 5. The dependence of the $\mathcal{R}$-statistic on the number of galaxies used to compute it. For simplicity, we assume here that the $\mathrm{BHG}$ is always the central, i.e. $f_{\mathrm{BNC}}=0$. The line-of-sight velocities of satellites are drawn from a single Gaussian. Note that the distribution of the $\mathcal{R}$-statistic has a slight dependence on $N_{\text {scd }}$ at fixed $f_{\mathrm{BNC}}$.

within a comoving distance corresponding to $z_{\max }=0.17$. For each galaxy we compute its apparent magnitude, taking into account $k$ - and evolution corrections as a function of redshift. The evolution correction has an analytical form, whereas we estimate the $k$-corrections by fitting a linear relation to the $k$-correction of observed galaxies in SDSS (Blanton \& Roweis 2007). We then require apparent magnitudes $m_{r}<17.6$, similar to the SDSS DR7 sample, and apply the SDSS DR7 survey mask. Additionally, we apply redshift space distortions according to the line-of-sight velocity of each galaxy. Furthermore, we add a random velocity drawn from a Gaussian with a scatter of $35 \mathrm{~km} \mathrm{~s}^{-1}$ to that line-of-sight velocity to account for spectroscopic redshift errors in the SDSS (More et al. 2009a). Finally, we simulate fibre collisions. If two galaxies are separated by less than $55^{\prime \prime}$ we assign one of them the redshift of the nearest neighbour with a probability of $65 \%$. This probability is not unity because some galaxy pairs are observed with several spectroscopic plates in which case redshifts can be measured for both despite the small separation.

\subsection{Parameter Inference}

We create 25 mock SDSS-like galaxy catalogues using the procedure described above. For a given choice of parameters, $\left\{f_{0}, a_{1}, a_{2}, b\right\}$, we can compute the $\mathcal{R}$ and $\mathcal{S}$-statistics in the mock catalogues. We divide our sample of primaries into a low, $z<0.09$, and a high, $z \geqslant 0.09$, redshift sample and 4 luminosity bins defined by 10.0,10.25, 10.5, 10.75 and 11.25 in $\log \left[L_{\mathrm{BHG}} /\left(h^{-2} \mathrm{~L}_{\odot}\right)\right]$. Altogether, we have 8 subsamples for which we compare the distributions of $\mathcal{R}$ and $\mathcal{S}$. For each of the 8 sub-samples the distributions of the $\mathcal{R}$ and $\mathcal{S}$-statistic are derived by merging the distributions of all 25 mock catalogues. Note that the $\mathcal{R}$ and $\mathcal{S}$-statistics at fixed $f_{\mathrm{BNC}}$ have a small dependence on the number of secondaries used. The dependence is such that a lower value of $N_{\text {scd }}$ has an effect similar to a higher value of $f_{\mathrm{BNC}}$. This is demonstrated in Figure 5 where we show the distribution of $\mathcal{R}$ values for different values of $N_{\text {scd }}$. For this plot, the velocities of secondaries were drawn from a single Gaussian, i.e. we assumed $f_{\mathrm{BNC}}=0$. This introduces the possibility that any miss-match in the $N_{\text {scd }}$ distributions between observations and our mock catalogues could bias our estimate of $f_{\mathrm{BNC}}{ }^{3}$ To alleviate the problem we weight each primary in the mock catalogues when calculating the $\mathcal{R}$ and $\mathcal{S}$ distributions. The weight for each primary is $N_{i, \text { obs }} / N_{i \text {,mock }}$ where $N_{i \text {,obs }}$ is the number of primaries with $i$ secondaries in the SDSS observations and $N_{i, \text { mock }}$ the same quantity for the mock surveys. We do this weighting in each of the 8 sub-samples separately. We note, however, that applying this weighting has only a very small influence on the resulting $\mathcal{R}$ and $\mathcal{S}$-distributions because $\left\langle N_{\mathrm{scd}}\right\rangle$ is overall well re-produced in the mocks.

To quantitatively compare the mock catalogues to the observations we fit binned distributions for both $|\mathcal{R}|$ and $|\mathcal{S}|$ in each of the 8 sub-samples. The bin edges are defined by 0 , $0.75,1.5,3.0$ and $\infty$. Under the assumption that all $\mathcal{R}$ and $\mathcal{S}$ values are independent, the probability mass function for a single sub-sample is given by a multinomial distribution such that the likelihood $\mathcal{L}$ is proportional to

$\mathcal{L} \propto \prod_{i=1}^{4} P_{i, \text { mock }}^{k_{i, \mathrm{obs}}}$,

where $P_{i \text {,mock }}$ is the probability that $|\mathcal{R}|$ or $|\mathcal{S}|$ are in a given bin as determined from the mock catalogues and $k_{i \text {,obs }}$ are the corresponding observed numbers.

We use MultiNest (Feroz \& Hobson 2008) to evaluate the parameter uncertainties. We assume a flat prior in $[-3,3]$ for $f_{0}$. On the other hand, $a_{1}, a_{2}$ and $b$ are generally not perfectly constrained and very large absolute values for these parameters are permitted by the data. We thus choose flat priors in $[-1,1]$ for $\hat{a}_{1}, \hat{a}_{2}$ and $\hat{b}$ defined by $a_{1}=\tan \left(\hat{a}_{1} \pi / 2\right)$, $a_{2}=\tan \left(\hat{a}_{2} \pi / 2\right)$ and $b=\tan (\hat{b} \pi / 2)$. This effectively maps $[-1,1]$ to $[-\infty, \infty]$. We use 1000 live points, a target sampling efficiency of $25 \%$ and $\Delta \ln \mathcal{Z}<0.1$ as a stopping criterion in all cases. Here, $\mathcal{Z}$ is the estimate for the global evidence (Feroz \& Hobson 2008). We have verified that our results are converged by running MultiNest with 2000 live points and using 50 mock catalogues and getting equivalent results. Altogether, roughly 60,000 likelihood evaluations are performed to arrive at the posterior distribution.

\section{RESULTS}

In this section we present our results from the analysis procedure described in the previous section.

\subsection{Default Analysis}

Figure 6 shows the distributions of the $\mathcal{R}$ and $\mathcal{S}$-statistics for the SDSS data (black with error bars), the mock catalogues with $f_{\mathrm{BNC}}=0$ for all halo masses and BHG luminosities (blue, dashed) and the best-fit, non-zero $f_{\mathrm{BNC}}$ model (red). We show all the 8 sub-samples divided into different BHG luminosities (different columns) and redshifts (different rows)

3 We have tested that using the biweight estimator (Beers et al. 1990) for $\hat{\sigma}_{\text {scd }}$ does not improve the situation. 


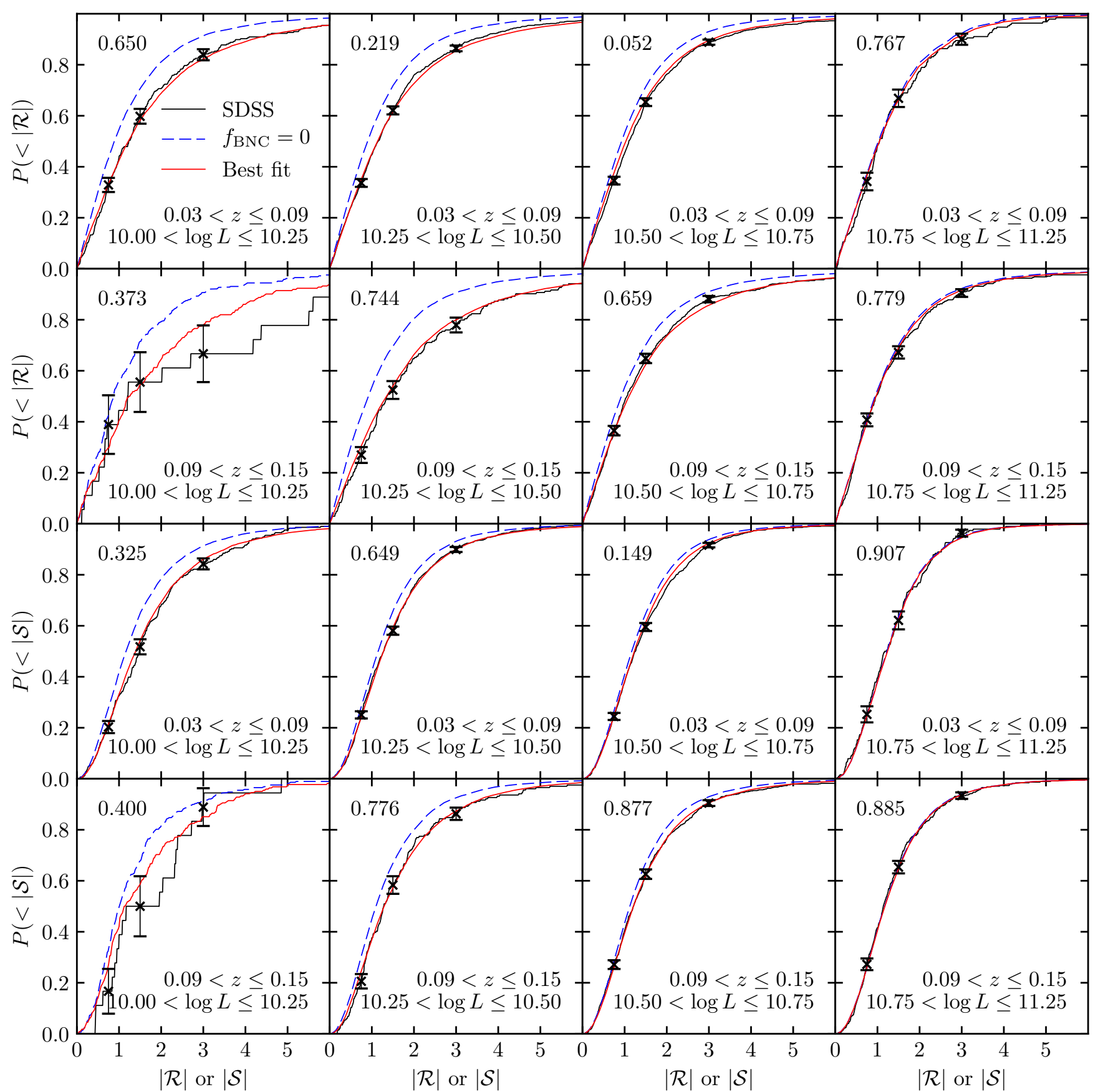

Figure 6. The $\mathcal{R}$ and $\mathcal{S}$ distributions for our mock catalogues with $f_{\mathrm{BNC}}=0$ (blue, dashed), the best-fitting model (red) and the SDSS data (black with error bars). The upper and lower two rows show the $\mathcal{R}$ - and $\mathcal{S}$-statistics, respectively. The luminosity increases from left to right. In the lower right corner of each plot we show the redshift range and BHG luminosity range in $\log \left[L_{\mathrm{BHG}} /\left(h^{-2} \mathrm{~L}_{\odot}\right)\right]$. Generally, the difference between the $f_{\mathrm{BNC}}=0$ mocks and the SDSS data decreases with luminosity. The best-fitting model can explain the data distribution in all cases. In the upper left side of each plot we show the $p$-value returned from a KS test comparing the best-fitting distributions of $|\mathcal{R}|$ or $|\mathcal{S}|$ against the SDSS measurements. The location of the error bars on the SDSS measurements correspond to the bin edges in $|\mathcal{R}|$ and $|\mathcal{S}|$ used for fitting the data.

that we used during the fitting, as indicated. The error bars on the SDSS data are computed assuming a binomial distribution, i.e. $\Delta P=\sqrt{P(1-P) / n}$, where $n$ is the number of primaries sampling the distributions. The $x$-positions of the error bars are the same as the bin edges used to fit the data. Note however that we used the binned probability distribu- tion function instead of the cumulative distribution function to fit the data.

The first thing to note is that the relative differences between the $f_{\mathrm{BNC}}=0$ model and the SDSS data decrease with increasing BHG luminosity. This indicates that $f_{\mathrm{BNC}}$ decreases with BHG luminosity for this particular sample of 
systems. Note however that this does not necessarily imply that $f_{\mathrm{BNC}}$, averaged over BHG luminosity, decreases with halo mass despite luminosity and halo mass being positively correlated. Instead, we will show later that there is a strong anti-correlation of $f_{\mathrm{BNC}}$ with BHG luminosity at fixed halo mass. This is the main driver of the apparent decrease of $f_{\mathrm{BNC}}$ with the luminosity of the BHG.

The best-fit model is able to accurately fit all 16 distributions. We can gauge the quality of the fit by using a Kolmogorov-Smirnov (KS) test to compare the best-fit $|\mathcal{R}|$ and $|\mathcal{S}|$ distributions to the SDSS data. The $p$-values of the KS-test are shown in the upper left corner of each plot. These $p$-values have not been used in the fit and we quote them here primarily to facilitate a comparison with van den Bosch et al. (2004) and Skibba et al. (2011) who also used KS tests. Most of the fits are excellent with $p$-values above $15 \%$. Alternatively, we can calculate a $\chi^{2}$ value by approximating all data points in the cumulative distribution function as a multi-variate Gaussian distribution. Under this approximation we get $\chi^{2} /$ dof $=35.1 /(48-4)$, again showing a good overall fit. Most importantly, our best-fit model can explain both the $\mathcal{R}$ and $\mathcal{S}$-distributions simultaneously for all BHG luminosities.

Our results are also relevant to the relative relaxation of the systems in our samples. As discussed in Skibba et al. (2011), if central galaxies would be unrelaxed with respect to the dark matter core, a scenario which we do not model, the effect on the $\mathcal{R}$-statistic would be much stronger than on the $\mathcal{S}$-statistic. Thus, our analysis confirms the findings of Skibba et al. (2011) that the detected phase-space offsets in the $\mathcal{R}$ and $\mathcal{S}$-statistics are likely due to satellites galaxies being BHGs.

The posterior distributions of the parameters are shown in Figure 7. The two-dimensional histograms show distributions for different parameter combinations with $68 \%$ and $95 \%$ confidence intervals. Note that we show $\hat{a}_{1}, \hat{a}_{2}$ and $\hat{b}$, not $a_{1}, a_{2}$ and $b$. The default analysis prefers models in which $n_{\mathrm{BTC}}$, and thereby $f_{\mathrm{BNC}}$, decreases with BHG luminosity at fixed halo mass $\left(a_{1}<0, a_{2}<0\right)$, in agreement with the visual inspection of Figure 6 , and increases with halo mass at fixed BHG luminosity $(b>0)$. Specifically, $b>0$ is found with $>98 \%$ probability. These constraints on $b$ primarily come from the fact that we divided our sample into a low and high-redshift sample in addition to splitting in BHG luminosity. Because the detection threshold increases with redshift and primaries need to have at least 3 detected secondaries, the high-redshift sample probes systems with higher halo masses on average compared to the low-redshift sample at the same BHG luminosity.

The main result of our work is summarized in Figure 8. We show $f_{\mathrm{BNC}}$ as a function of BHG luminosity for different halo masses. The bands indicate $68 \%$ confidence intervals and the dashed lines the distributions of BHG luminosities in our CLF models. Three different trends are apparent from this figure. Firstly, for a given halo mass the probability that the brightest galaxy is not the central strongly decreases with increasing BHG luminosity. This trend is expected because for a low-luminosity central galaxy it is more likely that its luminosity is exceeded by that of a satellite galaxy. Secondly, for a fixed BHG luminosity $f_{\mathrm{BNC}}$ increases with halo mass. This trend arises naturally from the fact that the satellite occupation or cluster richness increases with halo mass, making it more likely that a satellite is brighter than the central if the central luminosity is kept fixed. As we will show later, both qualitative results are also predicted by the CLF model of Cacciato et al. (2009, 2013). Finally, there is a trend of increasing $f_{\mathrm{BNC}}$ with halo mass in general. In particular, $f_{\mathrm{BNC}}$ for the median BHG luminosity increases with halo mass. We will discuss the trend of $f_{\mathrm{BNC}}$ with halo mass in more detail in section 6.1.

\subsection{Dependence on CLF Parameters}

Our results so far have been derived using mock catalogues constructed with a particular CLF parametrization. Specifically, we used the CLF parametrization and best-fit parameters of Cacciato et al. (2013). However, this model is an imperfect representation of the real Universe for several reasons. For example, the best-fit parameters in Cacciato et al. (2013) are not tuned to reproduce the observations that we consider, such as those of Figure 2. Also, Cacciato et al. (2013) fitted CLF and cosmological parameters simultaneously and the best-fit cosmological parameters are somewhat different from those adopted for the SMDPL simulations used to construct our mocks (see $\S 4$ ). Further, Cacciato et al. (2013) assumed a different, more concentrated radial profile for satellites to fit the clustering of galaxies in SDSS DR7. Finally, there are, of course, statistical and also some systematic uncertainties in the CLF parameters derived by Cacciato et al. (2013).

To test the impact of these systematic and statistical uncertainties on our results, we run our analysis procedure using mock catalogues with reasonable variations in the CLF parameters. These different variations are summarized in Table 2 . The first variation assumes a lower scatter in the central luminosity at a fixed halo mass. For this we leave all parameters unchanged but change the value for $\sigma_{\log L}$ from 0.157 dex to 0.120 dex. This value is roughly $5 \sigma$ away from the best-fit value found in Cacciato et al. (2013), but systematically lower values for $\sigma_{\log L}$ were inferred in analyses which omitted the Sloan Great Wall. In this case, the authors found $\sigma_{\log L}=0.142_{-0.009}^{+0.010}$. Thus, a value as low as 0.120 is plausible. The second variation has to do with the slope of the satellite CLF. Generally, the satellite CLF is given by Eq. (9), where $\alpha_{\mathrm{S}}$ is a free parameter in the analysis of Cacciato et al. (2013). This implies that the low-luminosity slope of the satellite CLF is independent of halo mass $M$. However, in an earlier paper Cacciato et al. (2009) introduced a parametrization with a variable luminosity slope,

$\alpha_{\mathrm{S}}(M)=-2.0+a_{1}\left(1-\frac{2}{\pi} \arctan \left[a_{2} \log \left(M / M_{2}\right)\right]\right)$,

where $a_{1}, a_{2}$ and $M_{2}$ are free parameters. A massindependent slope is realized by setting $a_{2}=0$. In this second variation of the CLF parameters we adopt the best-fit $a_{1}, a_{2}, M_{2}, b_{0}, b_{1}$ and $b_{2}$ from Cacciato et al. (2009) for the WMAP3 cosmology (Spergel et al. 2007). Note that Cacciato et al. (2009) used a different halo mass definition, but we do not apply a correction because the differences are small, typically $\sim 0.01$ dex. The last CLF parametrization assumes a lower overall number of satellites. To do this we change $b_{0}$ from -1.17 to -1.37 while leaving all other parameters unchanged. This is motivated by the finding that 


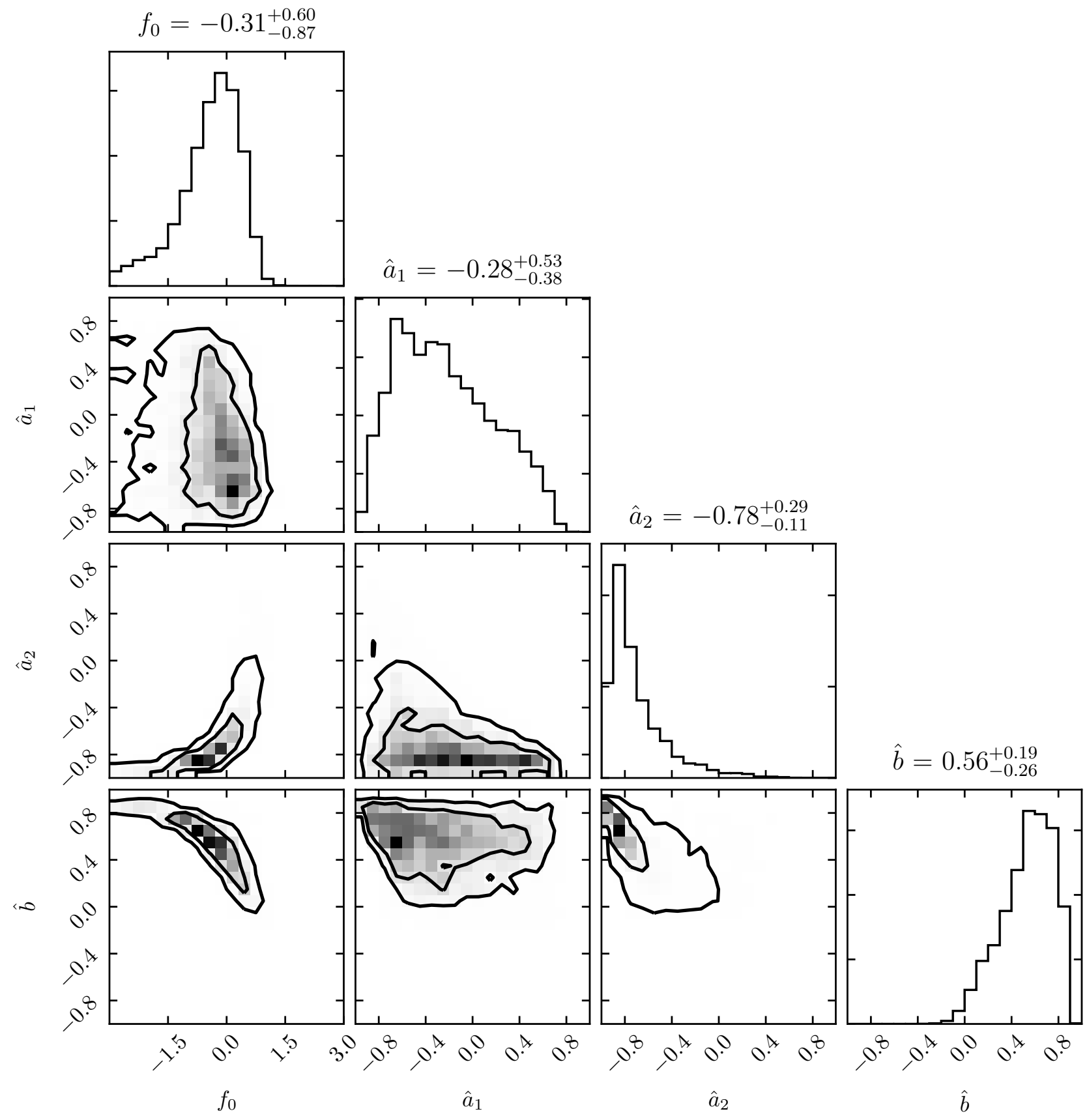

Figure 7. The marginalized posterior distributions of the parameters describing $f_{\mathrm{BNC}}\left(L_{\mathrm{BHG}}, M\right)$ for the default analysis. The model has been fitted to the entire SDSS sample. The contour levels on the two-dimensional histograms correspond to the $68 \%$ and $95 \%$ confidence intervals. We also show the marginalized one-dimensional posteriors for each parameter. Note that we show $\hat{a}_{1}, \hat{a}_{2}$ and $\hat{b}$, where $a_{1}=\tan \left(\hat{a}_{1} \pi / 2\right), a_{2}=\tan \left(\hat{a}_{2} \pi / 2\right)$ and $b=\tan (\hat{b} \pi / 2)$.

our mock catalogues produce more primaries with a given number of secondaries than in the SDSS.

We produce mock catalogues for all three CLF parametrizations and run the analysis in exactly the same way as for the default analysis. The marginalized posteriors for the parameters describing $f_{\mathrm{BNC}}$ are also listed in Table 2. Additionally, we show the posterior prediction for $f_{\mathrm{BNC}}$ as a function of halo mass and BHG luminosity for all CLF variations in Figure 9. We do not find that any of our qualitative results are impacted by alternative CLF choices. While some of the posteriors change slightly for different CLF models, none of the quantitative results change significantly. Only for the Fewer Satellites model we find that the best-fit $f_{\mathrm{BNC}}$ as a function of halo mass is roughly $5 \%$ lower. This is likely caused by this model producing a larger number of interlopers which have a similar effect as increasing $f_{\mathrm{BNC}}$. 


\begin{tabular}{ccccccccccc} 
Name & \multicolumn{9}{c}{ CLF Parameters } & \multicolumn{3}{c}{$f_{\text {BNC Posteriors }}$} \\
& $\sigma$ & $a_{1}$ & $a_{2}$ & $b_{0}$ & $b_{1}$ & $b_{2}$ & $f_{0}$ & $\hat{a}_{1}$ & $\hat{a}_{2}$ \\
\hline \hline Default & 0.157 & 0.82 & 0.00 & -1.17 & 1.53 & -0.217 & $-0.31_{-0.87}^{+0.60}$ & $-0.28_{-0.38}^{+0.53}$ & $-0.78_{-0.11}^{+0.29}$ & $0.56_{-0.26}^{+0.19}$ \\
Lower Sigma & 0.120 & 0.82 & 0.00 & -1.17 & 1.53 & -0.217 & $0.02_{-0.42}^{+0.41}$ & $-0.51_{-0.26}^{+0.45}$ & $-0.67_{-0.15}^{+0.38}$ & $0.50_{-0.21}^{+0.18}$ \\
Variable Slope & 0.157 & 0.50 & 2.11 & -0.77 & 1.01 & -0.094 & $0.07_{-0.44}^{+0.33}$ & $-0.44_{-0.33}^{+0.48}$ & $-0.62_{-0.19}^{+0.32}$ & $0.45_{-0.26}^{+0.24}$ \\
Fewer Satellites & 0.157 & 0.82 & 0.00 & -1.37 & 1.53 & -0.217 & $0.10_{-0.57}^{+0.45}$ & $-0.56_{-0.26}^{+0.50}$ & $-0.54_{-0.26}^{+0.52}$ & $0.50_{-0.32}^{+0.21}$ \\
\hline
\end{tabular}

Table 2. Parameters of the different CLF variations and their resulting marginalized posteriors for the parameters describing $f_{\mathrm{BNC}}$. All models have been fit to the entire SDSS sample. The 'Default' case represents the main result of our analysis.

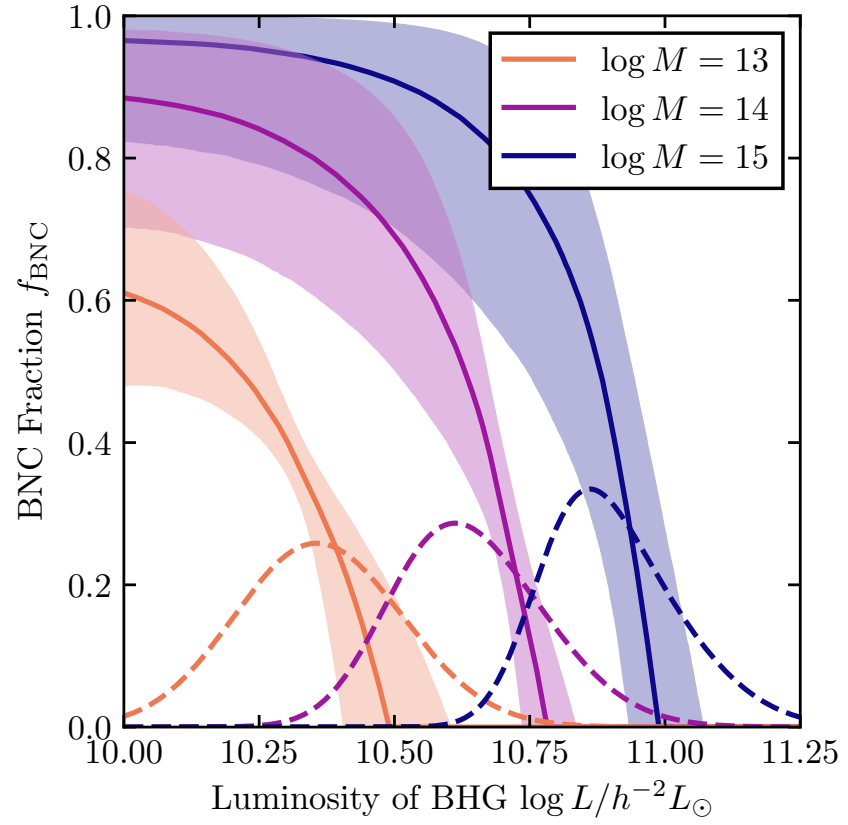

Figure 8. The posterior predictions for the fractions of haloes as a function of BHG luminosity where the brightest galaxy is not the central. Different colours indicate different halo masses in units of $\log \left[M_{200 b} /\left(h^{-1} \mathrm{M}_{\odot}\right)\right]$. Bands are $68 \%$ confidence intervals. The dashed lines show the distributions of BHG luminosities that result from our choice of the CLF model.

\section{DISCUSSION}

\subsection{Comparison to Previous Work}

Many previous studies have analysed the dynamical state of the BHGs (van den Bosch et al. 2005b; Sanderson et al. 2009; Zitrin et al. 2012). In particular, Skibba et al. (2011), Hikage et al. (2013), Wang et al. (2014) and Hoshino et al. (2015) have inferred $f_{\mathrm{BNC}}$ as a function of halo mass. To compare our result to these studies we marginalize the results shown in Figure 8 over the BHG luminosity distribution for each halo mass. The result is shown in Figure 10. Note that all studies use halo mass definitions slightly different from $M_{200 b}$, but we have not applied a correction because the differences are sufficiently small.

The analysis of Skibba et al. (2011) is very similar to our work. In particular, Skibba et al. (2011) used mock galaxy catalogues to fit the $\mathcal{R}$ and $\mathcal{S}$ distributions. The main difference is in their use of the Yang et al. (2007) group finder to identify galaxies belonging to the same halo and to measure halo masses. Additionally, Skibba et al. (2011) did not

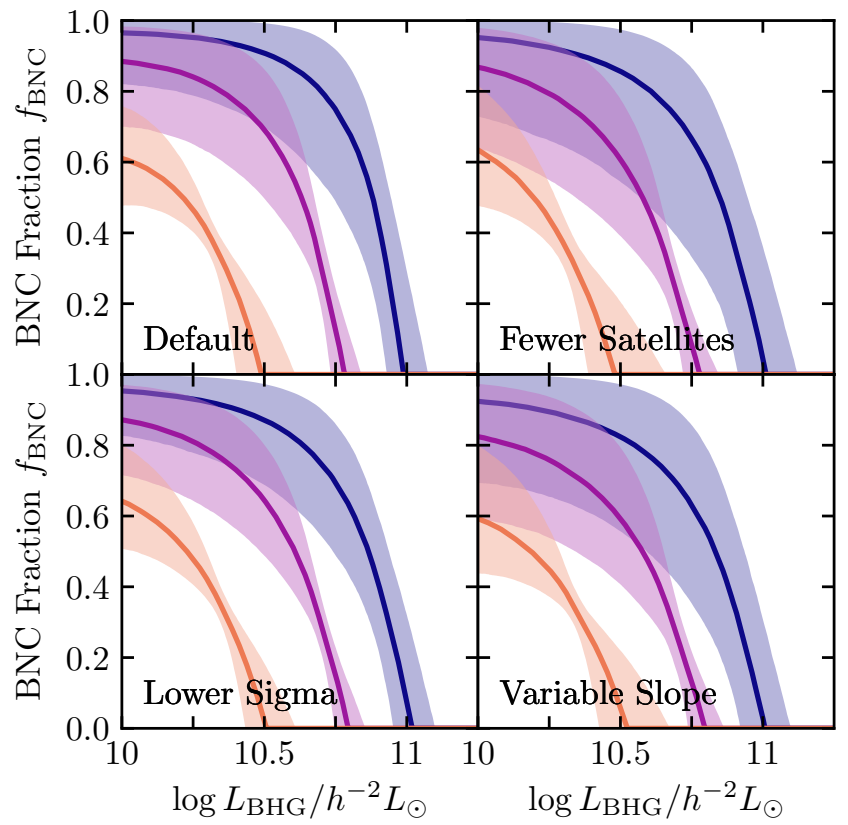

Figure 9. Similar to Figure 8, we show the posterior prediction for $f_{\mathrm{BNC}}$ as a function of BHG luminosity and halo mass. The colours have the same meaning as in Figure 8. Different panels are for different CLF choices to create the mock catalogues. All models have been fit to the entire SDSS sample.

characterize the dependence of $f_{\mathrm{BNC}}$ on BHG luminosity, and did not model several effects that we consider in this work, like the velocity offsets of halo cores, velocity correlations between satellites or the correlation of $n_{\text {sat }}$ and $p_{\mathrm{BNC}}$. Nevertheless, our results generally agree very well with those found by Skibba et al. (2011) at all halo masses. As discussed in section 4, we have improved upon the work of Skibba et al. (2011) by accounting for (i) velocity offsets between haloes and centrals, (ii) the unrelaxed state of the satellite population, (iii) correlation between $p_{\mathrm{BNC}}$ and the number of satellite galaxies, (iv) relaxing the assumption that satellite distributions are spherically symmetric and by (v) using a more conservative sample of primaries and secondaries. While many of these effects have a considerable impact on the $\mathcal{R}$ and $\mathcal{S}$ statistics individually (in many cases, the impact exceeds $1 \sigma$ significance), they happen to roughly cancel each other, such that our final constraints are very similar to those by Skibba et al. (2011). A more detailed analysis of the apparent agreement with Skibba et al. (2011) would involve a study of Yang et al. (2007) group finder errors and is beyond the scope of this work. 


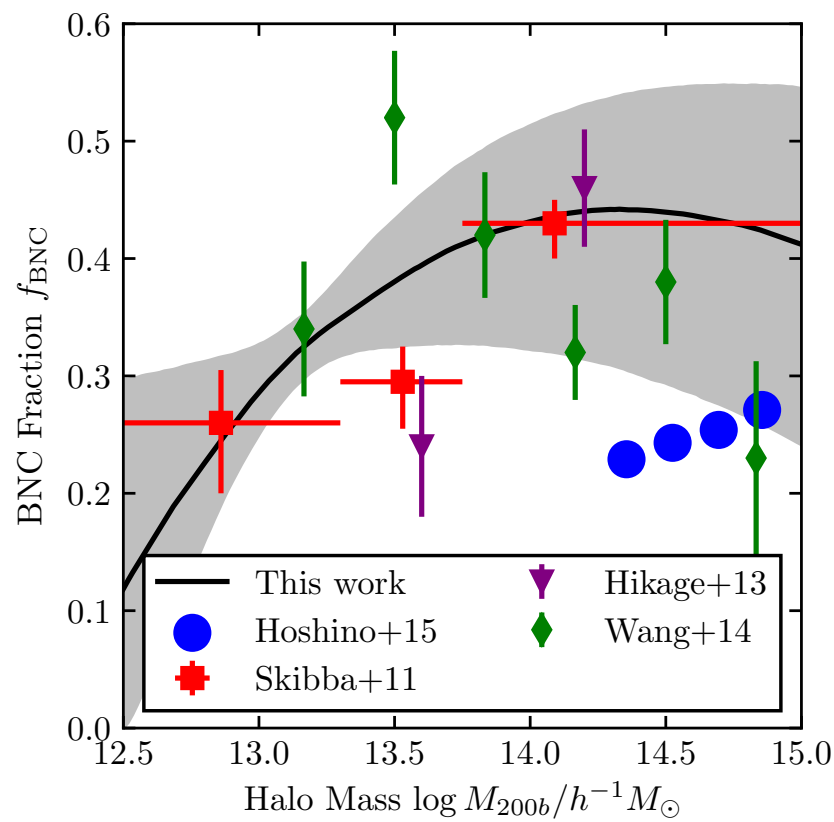

Figure 10. The fraction of systems in which the BHG is not the central as a function of halo mass. We compare our results (black, $68 \%$ confidence) to the findings of Skibba et al. (2011) (red squares), Hikage et al. (2013) (purple triangles), Wang et al. (2014) (green diamonds) and Hoshino et al. (2015) (blue dots). Note that Wang et al. (2014) determined how often the most massive galaxy is not the central whereas all other data points are for the most luminous. Error bars were added to the results of Wang et al. (2014) assuming a binomial distribution, i.e. $\Delta f_{\mathrm{BNC}}=\sqrt{f_{\mathrm{BNC}}\left(1-f_{\mathrm{BNC}}\right) / N}$, where $N$ is the number of groups analysed. The statistical uncertainties in the findings of Hoshino et al. (2015) were negligible and are not included in this figure. See the text for a detailed discussion of these results in the context of our findings.

$f_{\mathrm{BNC}}$ has been inferred for Luminous Red Galaxies (LRGs) from the SDSS DR7 (Hikage et al. 2013). The authors used the redshift-space power spectrum, LRG-galaxy weak lensing and the cross-correlation of LRGs with photometric galaxies to infer the fraction of off-centred LRGs. The analysis was done separately for systems with one LRG and for systems with multiple LRGs, as determined from a Counts-in-Cylinders technique. The strongest constraints for the off-centring fraction came from the crosscorrelation with photometric galaxies. It was found that $24 \pm 6 \%$ of LRGs in single-LRG systems and $46 \pm 5 \%$ of the brightest LRGs in multiple-LRG systems are off-centred, i.e. $f_{\mathrm{BNC}}=24 \pm 6 \%$ and $46 \pm 5 \%$ respectively. These values are shown in Figure 10 together with the average halo masses estimated from gravitational lensing (Hikage et al. 2013). Whereas the study by Hikage et al. (2013) focuses exclusively on haloes hosting LRGs as defined by the colour and magnitude cuts described in Eisenstein et al. (2001), our results are for all galaxies. Although it is non-trivial to compare results for these different selection criteria without a more detailed analysis, we speculate that the main impact is as follows; by restricting their analysis to systems that host at least one LRG, i.e. a galaxy with an absolute magnitude $M_{g}<-21.2$, the sample analysed by Hikage et al.
(2013) is likely biased towards systems with high $L_{\mathrm{BHG}}$ at fixed halo mass. Our analysis shows that these are systems for which $f_{\mathrm{BNC}}$ is relatively small. Particularly, we refer the reader to section 6.5 where we investigate $f_{\mathrm{BNC}}$ for haloes selected purely based on $L_{\mathrm{BHG}}$. Hence, we suspect that the results of Hikage et al. (2013) most likely represent a lower limit for the $f_{\mathrm{BNC}}$ of all galaxies. In light of this, and given the errors on their and our measurements, we conclude that the results presented by Hikage et al. (2013) are in reasonable agreement with those derived here.

Wang et al. (2014) studied the positions of X-ray peaks in clusters identified by the Yang et al. (2007) group finder in SDSS DR7. In their analysis, they denoted the central as the galaxy that is closest to the X-ray peak. Additionally, they required the central to be among the four most massive (in terms of stellar mass) galaxies in the cluster. They find that in roughly $35 \%$ of their halos the central defined in this way is different from the most massive galaxy. We show in Figure 10 the results for their sample of clusters with secure X-ray detections (Sample II) as a function of halo mass as determined from the Yang et al. (2007) group catalogue. Note that Wang et al. (2014) determined the fraction of haloes in which the most massive galaxy is not the central whereas we are concerned with the most luminous. However, in $\sim 90 \%$ of the groups the most luminous galaxy is also the most massive (Yang et al. 2008; Li et al. 2014). Overall, our findings are in good agreement with those found by Wang et al. (2014) using a completely different method. However, also note that the position of the X-ray peak does not necessarily always coincide with the halo centre (Cui et al. 2016).

The off-centring of BHGs from group centres in the Galaxy And Mass Assembly survey (GAMA, Driver et al. 2011) has been examined by Oliva-Altamirano et al. (2014). The authors find off-centring fractions ranging from 10 to $17 \%$ for halo masses of $10^{13}$ to $\sim 4 \times 10^{14}$ using the GAMA galaxy group catalogue (Robotham et al. 2011). However, the definition of the group centre in the group catalogue is different from the true dark matter halo centre. As described in Robotham et al. (2011), the group centre is determined in an iterative fashion. At each step, the centre of light of all group members is computed and the most distant group member is rejected in subsequent iterations. This is done until two members are left from which the brighter group member is determined to be the group centre. We have tested that this algorithm is strongly biased towards declaring the BHG the group centre even if the BHG is a satellite of the dark matter halo of that group. Thus, the results of Oliva-Altamirano et al. (2014) should be regarded as a lower limit on $f_{\mathrm{BNC}}$ with respect to the dark matter halo centre.

Hoshino et al. (2015) analysed $f_{\mathrm{BNC}}$ in the LRG SDSS sample using the redMaPPer cluster catalogue. Specifically, the redMaPPer cluster finding algorithm determines for each cluster member the probabilities that it is a true member (as opposed to an interloper) and that it is the central. Taken at face value, these can be used, in a straightforward manner, to determine for each cluster the probability that its brightest member is not the central. Hoshino et al. (2015) analysed the halo mass range $10^{14.5}-10^{15.5} h^{-1} \mathrm{M}_{\odot}$ and find $f_{\mathrm{BNC}} \approx 20 \%-25 \%$ for halo masses up to $10^{15.0} h^{-1} \mathrm{M}_{\odot}$. In the same halo mass range our results suggest $f_{\mathrm{BNC}} \approx$ 
$40 \pm 15 \%$. Thus, our results are different from the findings of Hoshino et al. (2015) at the $1.5 \sigma$ level. Note that Hoshino et al. (2015) rely entirely on the cluster membership and central probabilities determined by redMaPPer. Recently, $\mathrm{Zu}$ et al. (2017) have shown that the cluster membership probabilities in redMaPPer can be biased due to contamination from interloper galaxies, which could potentially impact the findings of Hoshino et al. (2015). On the other hand, Hikage et al. (2017) presented evidence supporting the accuracy of central probabilities in redMaPPer. Finally, the results of Hoshino et al. (2015) also depend on the richness-based halo mass estimates of redMaPPer. The authors have shown that their results for $f_{\mathrm{BNC}}$ can range from $\sim 10 \%$ to $\sim 40 \%$ if an additional dependence of the halo mass estimator on the central luminosity is factored in.

It is also possible that part of the discrepancy arises from the SDSS, as discussed in Hoshino et al. (2015). The SDSS DR7 pipeline significantly underestimates the luminosity of luminous extended galaxies, especially massive elliptical or cD galaxies (Abazajian et al. 2009; Aihara et al. 2011; Bernardi et al. 2013). This could lead to an increased fraction of $f_{\mathrm{BNC}}$ observationally. Hoshino et al. (2015) argue that part of the disagreement between their results (using SDSS DR8) and Skibba et al. (2011) (using SDSS DR4) might be attributed to this bias. However, using SDSS DR7 we mostly confirm the findings of Skibba et al. (2011) and the improvement in the bias going from DR7 to DR8 is 'subtle at best' (Aihara et al. 2011). Finally, while we have tested our results with respect to reasonable changes in the CLF parameters, they might also be affected by our choice of cosmology or the way we place satellites in dark matter haloes. For example, we made the choice of assigning the most luminous satellites the highest $M_{\text {peak }}$ subhaloes. Other proxies of satellite luminosity will results in different radial profiles (van den Bosch et al. 2016) and could also impact our inferences. Such an analysis is beyond the scope of this work.

\subsection{Comparison to SAMs}

Skibba et al. (2011) have shown that their inferred values for $f_{\mathrm{BNC}}$ as a function of halo mass are significantly higher than expected from theoretical models of galaxy formation. In particular, Skibba et al. (2011) showed that the predictions of the SAMs of Croton et al. (2006) and Monaco et al. (2007) are roughly a factor of two lower. Since our values for $f_{\mathrm{BNC}}$ are in good agreement with Skibba et al. (2011), those conclusions also hold for our analysis. In addition, we have investigated $f_{\mathrm{BNC}}\left(M_{\mathrm{vir}}\right)$ for the model of De Lucia \& Blaizot (2007) and find values decreasing from $\sim 18 \%$ to $\sim 10 \%$ when going from a halo mass of $10^{13}$ to $10^{15} h^{-1} \mathrm{M}_{\odot}$. These values are quite similar to those of Croton et al. (2006). Thus, the De Lucia \& Blaizot (2007) model also predicts substantially lower values for $f_{\mathrm{BNC}}$ than we infer in this work.

Hence, our findings might reveal an actual shortcoming of galaxy formation models. Skibba et al. (2011) argue that SAMs often adopt a dynamical friction time-scale that is too short. That would result in satellites being disrupted too early, particularly for the more massive satellites which should have the shortest dynamical friction timescales. This will boost the luminosity (and stellar mass) gap between central and brightest satellites, and thus reduce $f_{\mathrm{BNC}}$. How-

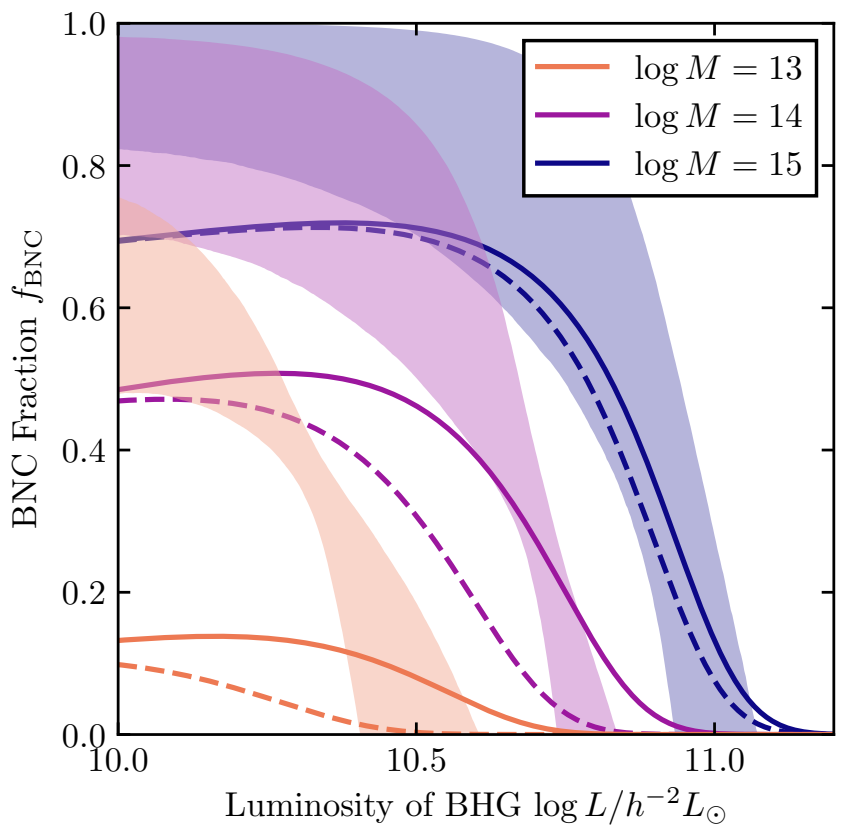

Figure 11. The fraction of systems in which the brightest galaxy is not the central as a function of BHG luminosity for different halo masses. Similar to Figure 8, bands show the $68 \%$ posterior prediction of our analysis using the whole SDSS sample. Dashed lines are the CLF predictions for our default model. Solid lines represent the CLF prediction from an optimized CLF model. See the text for details.

ever, note that De Lucia \& Blaizot (2007) use a dynamical friction time-scale that is twice the classical dynamical friction time-scale (Binney \& Tremaine 1987) used by Croton et al. (2006). Nevertheless, both produce similar predictions for $f_{\mathrm{BNC}}$. Other possibilities for an under-prediction of $f_{\mathrm{BNC}}$ could be an over-quenching of satellites (e.g., Weinmann et al. 2006) or an incorrect treatment of how centrals accrete stellar mass from disrupted satellites. It remains to be seen how other models, and hydrodynamical simulations of galaxy formation, fare in this respect.

\subsection{Implications for CLF Models}

For a given CLF model we can compute $f_{\mathrm{BNC}}$ as a function of $L_{\mathrm{BHG}}$ and $M_{200 b}$ via Eq. (12). In Figure 11 we show, similar to Figure $8, f_{\mathrm{BNC}}$ as a function of $L_{\mathrm{BHG}}$ for different halo masses. Bands shows the $68 \%$ posterior prediction from our analysis and the dashed lines are the predictions from the default CLF parametrization.

We first note that the CLF model reproduces the observations on a qualitative level. $f_{\mathrm{BNC}}$ increases with halo masses both in the CLF predictions and for our best-fit model. Also, the luminosity dependence for a fixed halo mass is quite similar in both cases. However, the CLF model persistently under-predicts $f_{\mathrm{BNC}}$, especially at lower halo masses. This is similar to the findings of Skibba et al. (2011). We conclude that our default CLF model, the one used in Cacciato et al. (2013), predicts a $f_{\mathrm{BNC}}\left(M, L_{\mathrm{BHG}}\right)$ that is incompatible with the constraints derived here. In the following, we discuss several possible reasons for this discrepancy. 
The CLF model of Cacciato et al. (2013) assumes that the central luminosity and the exponential cut-off luminosity of the satellite CLF have the same ratio for all masses, as given by Eq. (11). This ratio has not been fitted in the analysis of Cacciato et al. (2013), but has been fixed to the ratio found in Yang et al. (2008) from an analysis of the Yang et al. (2007) galaxy group catalogue. In that analysis, though, Yang et al. (2008) assumed that the most luminous (or most massive) galaxy is always the central and used this to decompose the CLF into a central and satellite component. In other words, the authors assumed $f_{\mathrm{BNC}}=0$. Thus, the low prediction for $f_{\mathrm{BNC}}$ from the CLF model might to some extent be a result of the input assumption of Yang et al. (2008).

Recently, Trevisan \& Mamon (2017) have analysed the statistics of magnitude gaps in the Yang et al. (2007) SDSS group catalogue. They found that the CLF parametrization of Yang et al. (2008) underpredicts the number of small-gap groups (SGG). They argue that a CLF model that allows for two central galaxies ${ }^{4}$ is in much better agreement with observations. While we have not analysed gap statistics, it is plausible that SGGs and groups where the BHG is not the central are closely connected.

It is also possible that some of the other intrinsic assumptions of CLF modelling are violated. One of those assumptions is that the central luminosity and the satellite CLF are independent at a fixed halo mass. However, we have shown that the SAM of De Lucia \& Blaizot (2007) predicts an anti-correlation between satellite occupation and central luminosity (cf. right-hand panel of Figure 4). Indeed, Hoshino et al. (2015) have argued that their results for the central occupation of redMaPPer clusters require such an anti-correlation in order to be reconciled with clustering studies. Furthermore, the authors state that preliminary studies of the CLF of redMaPPer clusters provides further evidence for such a scenario. Any kind of correlation between central luminosity and satellite occupation would certainly change the $f_{\mathrm{BNC}}$ prediction of CLF models. Similarly, a correlation between central and satellite luminosities could alter $f_{\mathrm{BNC}}$. Another common assumption of CLF models is that the satellite numbers follow a Poisson distribution. However, Jiang \& van den Bosch (2017) have shown that subhaloes, which are believed to host satellite galaxies, do not obey Poisson statistics (see also Boylan-Kolchin et al. 2010; Mao et al. 2015). Instead, the distribution of the mostmassive subhaloes is sub-Poissonian. If the same is true for the most luminous satellites, it might also have an impact on the $f_{\mathrm{BNC}}$ prediction of CLF models. It is also worth noting that neither of these effects have been modelled in our mock catalogues. Thus, they could also alter the derived values of $f_{\mathrm{BNC}}$ of our analysis.

If one wants to keep the traditional assumptions of CLF modelling, we can try to adjust our CLF parametrization to correctly predict $f_{\mathrm{BNC}}$. Particularly, we explore how changing the constant 0.25 in Eq. (9) changes the predictions of the CLF. One way would be to re-parametrize the satellite

4 We note that in our analysis a group can, by definition, only have one central.
CLF via

$\Phi_{\mathrm{s}}(L \mid M)=\frac{\Phi_{\mathrm{s}}^{\star}(M)}{L_{\star}}\left(\frac{L}{L_{\star}}\right)^{\alpha_{\mathrm{s}}} \exp \left[-\delta(M)\left(\frac{L}{L_{\star}}\right)^{2}\right]$,

where we have introduced

$\log \delta(M)=\delta_{1}+\delta_{2} \log \left[M_{200 b} /\left(10^{12} h^{-1} \mathrm{M}_{\odot}\right)\right]$,

a mass-dependent rescaling of the cut-off scale, and $\delta_{1}$ and $\delta_{2}$ as new free parameters. The original parametrization of Yang et al. (2007) is recovered for $\delta_{1}=\delta_{2}=0$. Experimenting with different values, we find that with $\delta_{1}=-0.5$ and $\delta_{2}=0.15$ the CLF predictions are in better agreement with the posterior predictions, as shown by the solid lines in Figure 11 (see also More 2012, for analogous conclusions obtained with a similar CLF modification to the one shown above). However, the CLF model is unable to reproduce the strong anti-correlation between $f_{\mathrm{BNC}}$ and $L_{\mathrm{BHG}}$ for low-mass haloes $\left(\sim 10^{13} h^{-1} \mathrm{M}_{\odot}\right)$. Even when setting $\delta(M)=0$, effectively removing the exponential cut-off, the strong increase of $f_{\mathrm{BNC}}$ with decreasing $L_{\mathrm{BHG}}$ at low halo masses is not achieved. In this case, the aforementioned anticorrelation between central luminosity and satellite occupation might be able to better explain this result.

To summarize, the best-fit model of Cacciato et al. (2013) underpredicts $f_{\mathrm{BNC}}$ as a function of BHG luminosity and halo mass. It remains to be seen how the CLF model needs to be modified in order to accommodate the high values of $f_{\mathrm{BNC}}\left(M, L_{\mathrm{BHG}}\right)$ inferred here. Most likely, such a modification will affect other statistics that depend on the CLF, such as galaxy clustering and galaxy-galaxy lensing. Whether a CLF model can be found that can simultaneously reproduce all these observables requires a joint analysis, which is beyond the scope of this work.

\subsection{Satellite Weak Lensing}

Gravitational lensing has been used successfully to measure the dark matter halo masses of satellite galaxies, both in the weak (e.g., Li et al. 2014; Sifón et al. 2015) and strong (e.g. Natarajan \& Kneib 1997; Natarajan et al. 2017) lensing regimes. Recently, several studies have used weak lensing to infer the stellar-to-halo mass ratio of satellite galaxies as a function of projected separation $r_{p}$ from the halo centre (Sifón et al. 2015; Li et al. 2016; Niemiec et al. 2017), finding evidence of tidal stripping near the halo centre.

The main observable in the above mentioned studies is the excess surface mass density profile $\Delta \Sigma(R)$ around satellite galaxies,

$\Delta \Sigma(R)=\bar{\Sigma}(<R)-\bar{\Sigma}(R)$,

where $R$ is the projected distance from the lens galaxy, $\bar{\Sigma}(<$ $R$ ) the average surface density for separations less than $R$ and $\bar{\Sigma}(R)$ the average surface density at $R$. If the lens is a satellite galaxy, the signal at small $R$ is dominated by the dark matter distribution of the subhalo and at large $R$ by the gravitational potential of the host halo. Satellite weak lensing studies rely on correctly identifying the group centre to accurately determine $r_{p}$ and also to not accidentally use a central galaxy as a lens. In the latter case, the excess surface mass density signal would be much larger at small scales due to the large gravitational potential of the halo centre. Some 


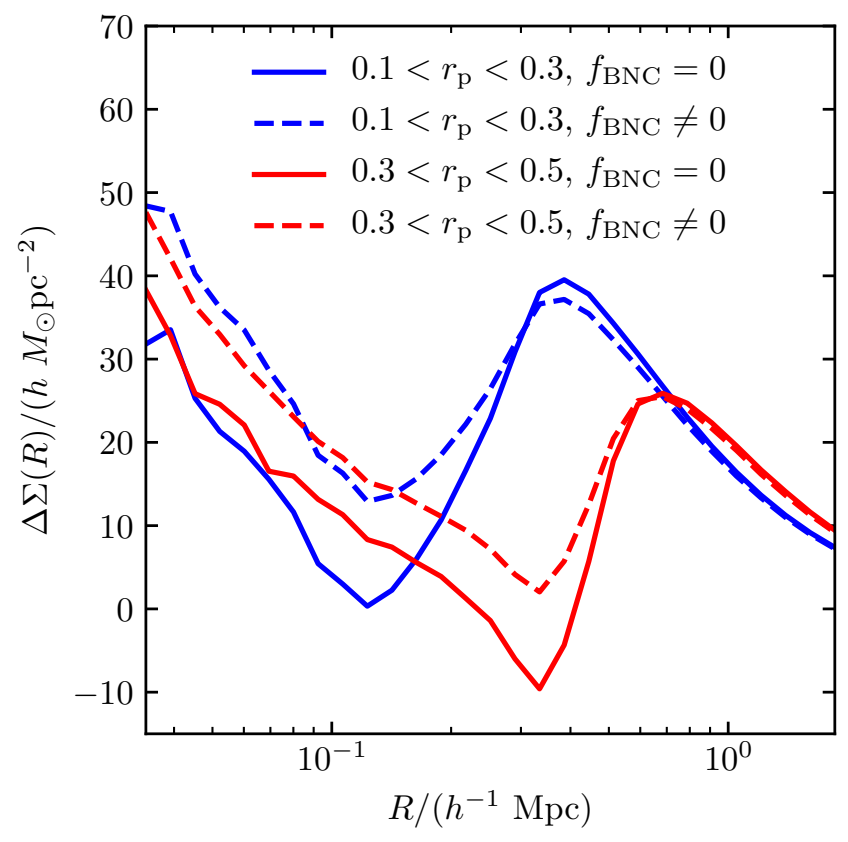

Figure 12. The excess surface mass density as a function of projected separation $R$ from the lens galaxy. For this plot we created a mock galaxy catalogue by populating the halo catalogue of the Bolshoi simulation according to our default CLF model. We concentrate on galaxies in host halos of masses $10^{13}-5 \times 10^{14} h^{-1} \mathrm{M}_{\odot}$. We split those galaxies by the projected separation to the $\mathrm{BHG}$, $0.1<r_{p} /\left(h^{-1} \mathrm{Mpc}\right)<0.3$ (blue) and $0.3<r_{p} /\left(h^{-1} \mathrm{Mpc}\right)<0.5$ (red). Additionally, we show predictions for a model with $f_{\mathrm{BNC}}=$ 0 (solid) and the best-fitting, non-zero model (dashed). In both cases, a non-zero $f_{\mathrm{BNC}}$ leads to an increase of the signal at small scales.

studies have used the BHG as the definition of the halo centre (Li et al. 2014; Sifón et al. 2015). Here, we test the impact of the resulting halo centre misidentification on the lensing signal.

We first populate the $z=0$ Bolshoi-Planck simulation (Riebe et al. 2011) with galaxies above a luminosity of $10^{9.7} h^{-2} \mathrm{~L}_{\odot}$ according to the procedure outline in section 4. This luminosity threshold roughly corresponds to the threshold used in Li et al. (2014). We further limit this analysis to galaxies living in halos of masses $10^{13} h^{-1} \mathrm{M}_{\odot}<$ $\mathrm{M}_{\text {vir }}<5 \times 10^{14} \mathrm{~h}^{-1} \mathrm{M}_{\odot}$. For each galaxy we calculate the projected distance $r_{p}$ to the BHG in the $x y$-plane. Next, we follow $\mathrm{Li}$ et al. (2014) and bin the galaxies into two bins of projected separation; $0.1<r_{p} /\left(h^{-1} \mathrm{Mpc}\right)<0.3$ and $0.3<r_{p} /\left(h^{-1} \mathrm{Mpc}\right)<0.5$. Finally, we use the BolshoiPlanck particle catalogue and the delta_sigma function provided by halotools to calculate the lensing signal around galaxies in each bin of projected separation. We do this both for a model with $f_{\mathrm{BNC}}=0$ and for the best-fitting, non-zero $f_{\mathrm{BNC}}$ model.

In Figure 12 we show the excess surface mass density as a function of projected separation from the lens galaxy for all 4 samples. We find that a non-zero $f_{\mathrm{BNC}}$ leads to small changes at large projected radii from the lens and to a systematically higher excess surface mass density at small separations. Thus, not taking a non-zero $f_{\mathrm{BNC}}$ into account would likely lead to an overestimation of the average subhalo mass. However, the effect seems to be mostly independent of the separation $r_{p}$ of the lens galaxy from the BHG. Furthermore, the strength of the effect is below current statistical uncertainties. Thus, it seems unlikely that a non-zero $f_{\mathrm{BNC}}$ would lead to wrong inferences about tidal stripping of galaxies (Sifón et al. 2015; Li et al. 2016; Niemiec et al. 2017) or subhalo segregation (van den Bosch et al. 2016). Our results for the impact of a non-zero $f_{\mathrm{BNC}}$ on the weak lensing signal of satellite galaxies are in agreement with $\mathrm{Li}$ et al. (2014).

\subsection{Impact on Satellite Kinematics}

Satellite kinematics studies similar to the ones performed by van den Bosch et al. (2004) and More et al. (2011) are a promising avenue to infer the galaxy-halo connection from small-scale redshift space distortions (see also e.g., Prada et al. 2003; Conroy et al. 2007; Norberg et al. 2008; Li et al. 2012; Wojtak \& Mamon 2013). These studies utilize a similar isolation criteria as used in this study and measure the pairwise velocity dispersion between primaries and their secondaries as a function of the luminosity of the primary. The velocity dispersion, in turn, is a measure of the halo mass. Generally, the latest results of More et al. (2011) are in good agreement with independent studies using galaxygalaxy lensing, group catalogues or galaxy clustering. However, More et al. (2011) acknowledge that their study seems to lead to systematically higher values for the inferred average halo masses. Importantly, when comparing their model to the data, More et al. (2011) assume that the BHG is always the central. Clearly, if the BHG were not the central, the pairwise velocity dispersion between the primary and secondaries will be higher, simply because satellites have larger peculiar velocities relative to their halo than centrals. This could, in principle, explain part of the discrepancy identified by More et al. (2011) (see also Dutton et al. 2010). This was previously discussed in Skibba et al. (2011) and More et al. (2011) who estimated that this effect could lead to systematic errors in halo mass of up to a factor of 1.6. However, to estimate the impact of this effect on satellite kinematics precisely one needs to know $f_{\mathrm{BNC}}$ as a function of both halo mass and BHG luminosity. This information was not available previously, but with the results of this study we can asses the impact quantitatively.

To study this effect we first populate the SMDPL $z=0.1$ halo catalogue with galaxies according to the recipe described in section 4 and a luminosity threshold of $10^{9.5} h^{-2} \mathrm{~L}_{\odot}$. This is the same threshold as used by More et al. (2011). We want to explore the effect of a non-zero $f_{\mathrm{BNC}}$ without any observational complications. Thus, we do not include the projection onto the sphere, the SDSS survey mask, magnitude thresholds or fibre collisions. Instead, we simply apply our isolation criteria in the distant observer approximation, i.e., using the simulation z-axis as the lineof-sight direction. The parameters of the cylinder are now $(\Delta V)_{\mathrm{h}}=5 \sigma,(\Delta V)_{\mathrm{s}}=4000 \mathrm{~km} \mathrm{~s}^{-1}, R_{\mathrm{s}}=0.15 \sigma_{200} h^{-1} \mathrm{Mpc}$ and $R_{\mathrm{h}}=5.33 R_{\mathrm{s}}$, the same as in More et al. (2011). We also remove secondaries which do not belong to the same halo as the primaries, and primaries without secondaries. Finally, we measure as a function of the BHG luminosity the average number of secondaries which More et al. (2011) denote by $\left\langle N_{\text {sat }}\right\rangle$ and the average pairwise velocity dispersion between 


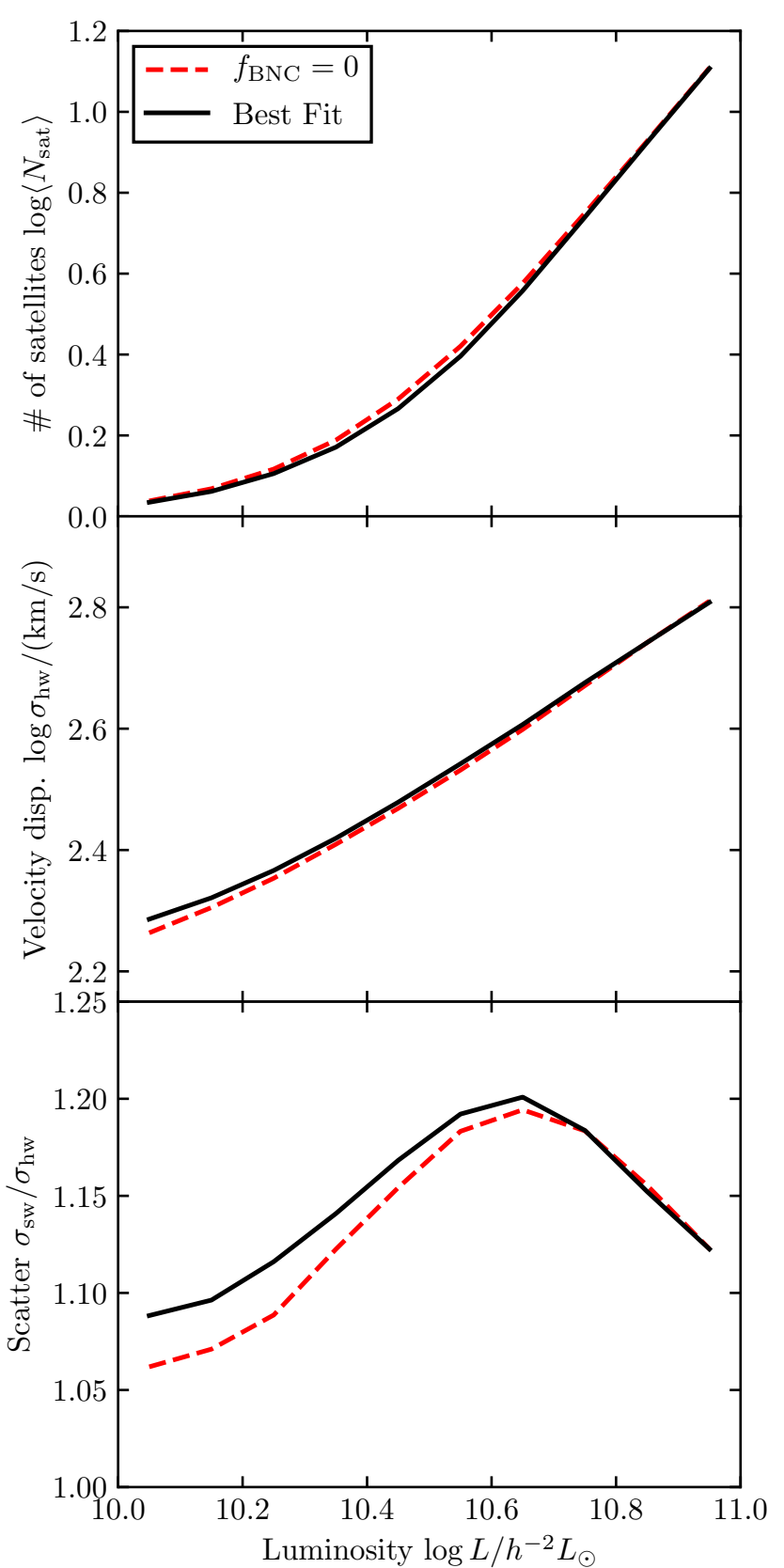

Figure 13. The impact of a non-zero $f_{\mathrm{BNC}}$ on the observables in satellite kinematic studies similar to More et al. (2011). In each panel we compare the predictions from a model in which all BHGs are central (red, dashed) to the best-fitting, non-zero $f_{\mathrm{BNC}}$ model (black, solid). Both models otherwise assume the same CLF parameters and were applied to the SMDPL ROCKSTAR halo catalogue. The upper panel shows the average number of secondaries around primaries. The middle panel displays the average velocity dispersions as a function of primary luminosity. Finally, the lower panel shows the ratio of satellite-weighted to host-weighted velocity dispersion, effectively a measure of the scatter in halo mass at fixed luminosity. Clearly, a non-zero $f_{\mathrm{BNC}}$ similar to that inferred here has only a very mild impact on studies of satellite kinematics. the primary and its secondaries. As discussed in More et al. (2009b), the average velocity dispersion can be obtained by either giving each primary a weight of unity (host-weighted, $\sigma_{\mathrm{hw}}$ ) or by giving each primary a weight proportional to the number of secondaries around it (satellite-weighted, $\sigma_{\mathrm{sw}}$ ). Generally, the satellite-weighted velocity dispersion estimate is expected to be higher because primaries with more secondaries tend to reside in more massive haloes. Thus, the ratio $\sigma_{\mathrm{sw}} / \sigma_{\mathrm{hw}}$ is a measure of the scatter in halo mass for primaries of the same luminosity.

Figure 13 shows the effect of the best-fit $f_{\mathrm{BNC}}$ versus $f_{\mathrm{BNC}}=0$ on these observables. Both models otherwise use the exact same CLF. In each panel, the red dashed line shows the result if all BHGs were centrals and the black solid line the result of our best-fitting model. The upper panel shows the average number of secondaries as a function of BHG luminosity. As expected, the number of secondaries scales with the luminosity of the primary. The best-fitting model predicts a slightly lower $\left\langle N_{\text {sat }}\right\rangle$. This is expected because we only assign galaxies within a certain projected radius around the primary galaxy. If the primary is a satellite that is far away from the centre of the halo, the expected number of associations is smaller. The middle panel shows the impact on the host-weighted velocity dispersion. Generally, more luminous galaxies have higher velocity dispersions. As expected, the pairwise velocity dispersion is higher for our best-fit model compared to the $f_{\mathrm{BNC}}=0$ case. However, the effect is small, $\sim 3 \%$ at most for $\log L / h^{-2} \mathrm{~L}_{\odot}>10.2$. One reason for this, as we will show below, is that $f_{\mathrm{BNC}}$ as a function of BHG luminosity, averaged over halo mass, is very low. Additionally, the velocity correlation between satellites also decreases the measured velocity dispersion if a satellite is selected as a primary. The systematic impact on halo mass, using $M \propto \sigma^{3}$, is at most $\sim 10 \%$. This is below the statistical uncertainties in More et al. (2011). Finally, the lower panel shows the ratio $\sigma_{\mathrm{sw}} / \sigma_{\mathrm{hw}}$. Our best-fit model predicts a slightly higher ratio for the same CLF parameters. This makes sense because at a given BHG luminosity halo mass and $f_{\mathrm{BNC}}$ have a positive correlation. Thus, $\sigma_{\mathrm{sw}}$ being dominated by high-mass haloes is affected more strongly by a non-zero $f_{\mathrm{BNC}}$. In principle, this could lead to an overestimate of the scatter in central luminosity as a function of halo mass if not accounted for. But, again, the effect seems to be too small to significantly affect the results of More et al. (2011).

One reason for the low impact of the best-fitting, nonzero $f_{\mathrm{BNC}}$ model on satellite kinematic observables is that $f_{\mathrm{BNC}}$ as a function of BHG luminosity is much lower than as a function of halo mass. This is shown in Figure 14 where we show $f_{\mathrm{BNC}}$ as a function of BHG luminosity averaged over all halo masses in SMDPL. The grey band is the $68 \%$ posterior of our default analysis and the solid line the best-fit model. We see that $f_{\mathrm{BNC}}$ has only a weak dependence on BHG luminosity and does not exceed $\sim 15 \%$. This might seem at odds with Figure 6 , which suggests that $f_{\mathrm{BNC}}$ decreases strongly with $L_{\mathrm{BHG}}$. However, for our sample in Figure 6 we did require at least 3 detected secondaries. That means that at the low-luminosity end this sample is biased towards highmass haloes, those with high $f_{\mathrm{BNC}}$. Overall, the reason for the low $f_{\mathrm{BNC}}$ is that the halo mass function is relatively steep and that there is scatter in BHG luminosity at fixed halo mass. That implies that the number density of galaxies at a 


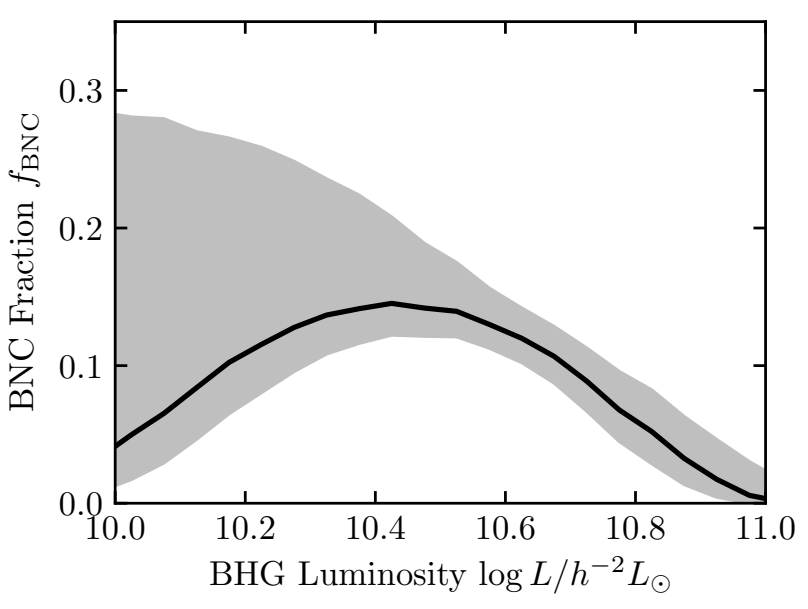

Figure 14. The inferred $f_{\mathrm{BNC}}$ as a function of BHG luminosity, marginalized over the halo mass function. The solid line represents the median of the posterior while the grey band denotes the $68 \%$ uncertainty on the posterior prediction.

given BHG luminosity is dominated by low-mass haloes with over-luminous BHGs compared to more massive haloes with under-luminous BHGs. Coincidently, we find that low-mass haloes with high-luminosity BHGs have very low $f_{\mathrm{BNC}}$. Note also that these conclusions are to some extent dependent on the extrapolation of our $f_{\mathrm{BNC}}$ parametrization to lower halo masses. This is signified by the large error bars at the lowluminosity end in Figure 14. However, we have tested that even the $15\left\{f_{0}, a_{1}, a_{2}, b\right\}$ combinations from our posterior with the lowest likelihood, $\Delta \ln \mathcal{L} \sim 10$ compared to the best-fit model, do not change the above conclusions.

We conclude that our results for $f_{\mathrm{BNC}}$ imply a very small impact on satellite kinematic studies (van den Bosch et al. 2004; More et al. 2009a, 2011; Li et al. 2012). Essentially, assuming $f_{\mathrm{BNC}}=0$ seems to be a good approximation given current statistical uncertainties.

\subsection{Observed Radial Profile}

One crucial assumption going into the modelling of galaxy clustering, galaxy-galaxy lensing and satellite kinematics is the radial profile of satellite galaxies (see, e.g. More et al. 2009a; Guo et al. 2012, 2013). Evidently, the radial profile has a direct impact on the observed galaxy-galaxy and galaxy-matter correlation. Furthermore, satellite kinematic studies infer halo masses by modelling the dynamics using the Jeans equation. This calculation needs the radial profile of satellite galaxies as an input. The effect of different assumed radial profiles on the predicted velocity dispersion are considerable. For example, if subhaloes were anti-biased with respect to the dark matter, the expected velocity dispersion would be higher by up to 20\% (van den Bosch et al. 2004).

More et al. (2009a) derived the radial profile of satellite galaxies using observations and concluded that satellite galaxies were anti-biased with respect to the dark matter distribution. However, this result was derived assuming that all BHGs were centrals. The concern is that a non-zero

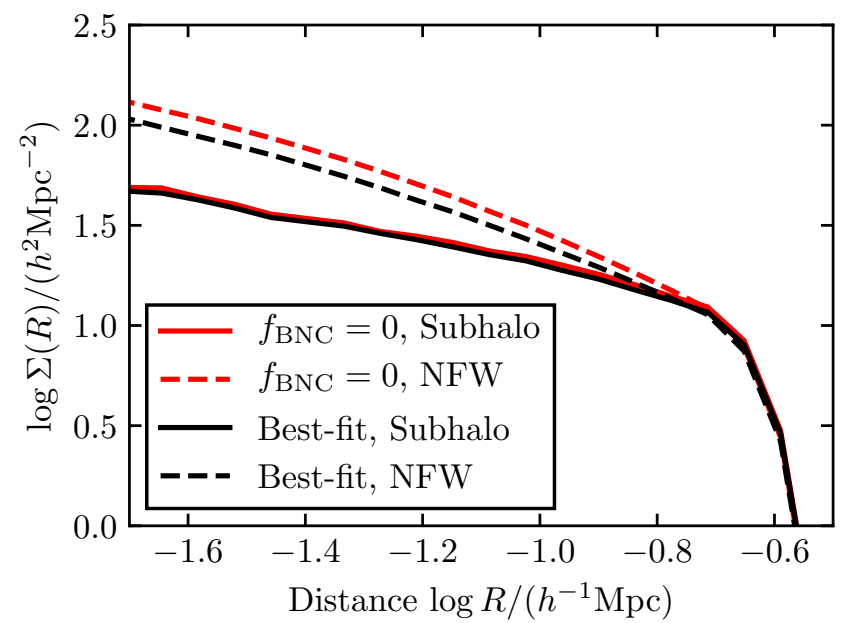

Figure 15. The surface density of secondaries around primaries with $10.5<\log \left[L /\left(h^{-2} L_{\odot}\right)\right]<10.7$ in mock catalogues. The black lines show results from our best-fit, non-zero $f_{\mathrm{BNC}}$ model and the red lines the results for $f_{\mathrm{BNC}}=0$. Conversely, solid lines denote mock catalogues where the satellite phase-space positions are modelled after subhaloes in SMDPL, while dashed lines represent simulations where satellites are assumed to follow an NFW profile. Note that, in both cases, the best-fit model and the model with $f_{\mathrm{BNC}}=0$ yield inferred surface densities that are virtually indistinguishable. Hence, a non-zero $f_{\mathrm{BNC}}$ similar to that inferred here does not have a significant impact on the inferred surface density profile of satellite galaxies.

$f_{\mathrm{BNC}}$ would systematically alter the observed radial profile. In general, if some satellite galaxies are BHGs, and consequently mis-classified as centrals, the inferred radial profile will be less centrally concentrated. In the case where satellite galaxies are an un-biased tracer of the underlying mass distribution, this will result in the incorrect conclusion that satellites are anti-biased with respect to their dark matter halo.

We address this issue in Figure 15 where we show the measured surface density of secondaries around primaries with $\log \left[L /\left(h^{-2} \mathrm{~L}_{\odot}\right)\right] \in[10.5,10.7]$. The solid lines are similar to the ones in Figure 13, showing the different observed radial profiles for $f_{\mathrm{BNC}}=0$ and the best-fitting non-zero $f_{\mathrm{BNC}}$ model. In particular, for this model we assume that satellites follow the subhalo distribution. As in the previous figure, the effect of interlopers, fibre collisions, projection effects etc., have been ignored. The down-turn at high radii simply reflects the radius $R_{\mathrm{s}}$ of the cylinder used to associate secondaries with primaries. Again, the impact of a non-zero $f_{\mathrm{BNC}}$ is negligible. There is virtually no difference between the observed profiles. On the other hand, the dashed lines show the predictions if satellite galaxies would follow an NFW profile with the same concentration as the dark matter distribution. For this model we assigned phase-space coordinates using the NFWPhaseSpace function of halotools. As shown in Figure 15, even in the case of a very steep radial profile like NFW, the best-fit, non-zero $f_{\mathrm{BNC}}$ does not significantly impact the observed surface number density profile of satellite galaxies around BHGs. 


\section{CONCLUSION}

Theoretical models predict that central galaxies of dark matter haloes should constitute a special population of halo members that are more luminous than satellite galaxies. Thus, it is commonly assumed that the BHG of a dark matter halo is the central. In this paper we have analysed data from the NYU-VAGC (Blanton et al. 2005) built upon SDSS DR7 (Abazajian et al. 2009). In particular, we investigated the phase-space positions of BHGs with respect to galaxies living in the same halo. Following van den Bosch et al. (2004) and Skibba et al. (2011) we computed the $\mathcal{R}$ and $\mathcal{S}$ statistic for a sample of galaxies with particularly high probabilities of being BHGs. These quantities measure velocity and spatial offsets, respectively. We compare this to detailed mock catalogues to model the fraction $f_{\mathrm{BNC}}$ of haloes in which the BHG is not the central as a function of both halo mass and BHG luminosity. Compared to Skibba et al. (2011) our model contains several improvements.

- Central galaxies are assigned the phase-space positions of halo cores instead of the bulk velocity of the entire halo. This accounts for the unrelaxed state of the dark matter distribution of the halo. Since the effect of phase-space offsets of the central and an increased $f_{\mathrm{BNC}}$ are qualitatively similar (Skibba et al. 2011), this is important to get an unbiased estimate of $f_{\mathrm{BNC}}$.

- We assign phase-space positions for satellite galaxies using the positions of resolved subhaloes. Compared to Skibba et al. (2011) this explicitly models the effects of satellite phase-space correlations and halo triaxiality.

- As described in section 4.4, our model takes into account a correlation between satellite occupation and the probability that the brightest galaxy is not the central. This is important because our sample is biased towards systems with larger satellite occupation.

We model the $\mathcal{R}$ and $\mathcal{S}$ distributions in 4 bins of BHG luminosity and 2 redshift bins. Our best-fit model for $f_{\mathrm{BNC}}$ as a function of halo mass and BHG luminosity is able to explain the observed $\mathcal{R}$ and $\mathcal{S}$ distributions at all BHG luminosities ranging from $10^{10}$ to $10^{11.25} h^{-2} L_{\odot}$ and redshifts ranging from 0.03 to 0.15 . We find that for a given halo mass, $f_{\mathrm{BNC}}$ is strongly anti-correlated with the luminosity of the BHG. Such an anti-correlation is expected from theoretical models of galaxy formation and halo occupation models like the CLF. This effect can be understood by the fact that it is more likely that a satellite exceeds the luminosity of the central if the latter one is low. At a fixed BHG luminosity, the probability that the BHG is not the central is increasing with halo mass. Again, this outcome is in agreement with predictions from CLF models and naive expectations based on the increasing satellite occupation with halo mass. Furthermore, $f_{\mathrm{BNC}}$ marginalized over the BHG luminosity distribution of each halo increases with halo mass. Comparing our results to previous studies, we find that $f_{\mathrm{BNC}}$ as a function of halo mass is in good quantitative agreement with the findings of Skibba et al. (2011) and in slight tension with the results of Hoshino et al. (2015).

Similar to Skibba et al. (2011), we find that our inferred values for $f_{\mathrm{BNC}}$ are surprisingly high compared to the SAMs of Croton et al. (2006), De Lucia \& Blaizot (2007) and Monaco et al. (2007) and compared to naive 'predictions' based on published CLF models. We find values for $f_{\mathrm{BNC}}$ ranging from $25 \%$ to $40 \%$ in the halo mass range $10^{13}$ to $10^{15} h^{-1} M_{\odot}$, whereas the SAMs and CLF generally predict values below $20 \%$. This disagreement could arise from inaccuracies in the SDSS photometric pipeline (Abazajian et al. 2009; Aihara et al. 2011) or might signal an actual shortcoming of the theoretical models. For example, it is possible that SAMs suffer from over-merging or over-quenching of satellite galaxies. It is also possible that certain common assumptions of CLF modelling, such as the statistical independence of central and satellite occupation, are violated. It will be interesting to extend our analysis to lower luminosities and halo masses, which may be possible with surveys such as GAMA (Driver et al. 2011).

Using our best-fit model for $f_{\mathrm{BNC}}$ we explore the impact of a non-zero $f_{\mathrm{BNC}}$ onto studies that implicitly assume that the BHG is the central. Satellite kinematic studies (e.g., van den Bosch et al. 2004; Conroy et al. 2007; Norberg et al. 2008; More et al. 2009b,a, 2011; Li et al. 2012) infer the average halo mass of an ensemble of BHGs by modelling the redshift-space correlations with other halo members. Typically, in order to model the inferred halo masses it is explicitly assumed that the BHG is always the central, i.e. $f_{\mathrm{BNC}}=0$. Here, we have shown that this simplification only leads to a very modest bias in the inferred halo masses of order $\lesssim 10 \%$. We also explored the impact of assuming $f_{\mathrm{BNC}}=0$ on the observed radial profile of satellite galaxies around BHGs. Once again, we find that the impact is subtle at most. Most importantly, it is unlikely that the inference that satellite galaxies are strongly anti-biased (i.e., less centrally concentrated) with respect to the dark matter distribution (e.g., Yang et al. 2005; Chen 2008; More et al. 2009a), is solely caused by this assumption. Finally, our best-fit models allow to test the impact of this assumption for other observables such as galaxy-clustering and galaxygalaxy lensing. Many of the findings of the present analysis will also be used in a future work employing satellite kinematics and gravitational lensing.

\section{ACKNOWLEDGEMENTS}

This research was supported in part by the National Science Foundation under Grant No. NSF PHY-1125915. JUL was supported by a KITP graduate fellowship. FvdB and JUL are supported by the US National Science Foundation through grant AST 1516962. APH is funded through the Yale Center for Astronomy \& Astrophysics Prize fellowship. ARZ is funded by the Pittsburgh Particle physics Astrophysics and Cosmology Center (Pitt PACC) at the University of Pittsburgh and by the NSF through grant NSF AST 1517563. YYM is supported by the Samuel P. Langley PITT PACC Postdoctoral Fellowship. This work was supported by the HPC facilities operated by, and the staff of, the Yale Center for Research Computing.

This work made use of the following software packages: matplotlib (Hunter 2007), SciPy, NumPy (van der Walt et al. 2011), Astropy (Astropy Collaboration 2013), Cython (Behnel et al. 2011), halotools (Hearin et al. 2016), Corner (Foreman-Mackey 2016), MultiNest (Feroz \& Hobson 2008; Feroz et al. 2009, 2013), PyMultiNest (Buchner et al. 2014), mangle (Hamilton \& Tegmark 2004; Swanson et al. 2008) 
and pymangle $e^{5}$. We are very grateful to all contributors to the above mentioned software packages which helped to greatly expedite this work. We thank the anonymous referee for an insightful report that significantly improved the presentation of this paper. We are also grateful to Alexie Leauthaud, Surhud More, Sarah Brough and Marina Trevisan for their helpful discussions.

Funding for the Sloan Digital Sky Survey (SDSS) has been provided by the Alfred P. Sloan Foundation, the Participating Institutions, the National Aeronautics and Space Administration, the National Science Foundation, the U.S. Department of Energy, the Japanese Monbukagakusho, and the Max Planck Society. The SDSS Web site is http://www.sdss.org/.

The SDSS is managed by the Astrophysical Research Consortium (ARC) for the Participating Institutions. The Participating Institutions are The University of Chicago, Fermilab, the Institute for Advanced Study, the Japan Participation Group, The Johns Hopkins University, Los Alamos National Laboratory, the Max-Planck-Institute for Astronomy (MPIA), the Max-Planck-Institute for Astrophysics (MPA), New Mexico State University, University of Pittsburgh, Princeton University, the United States Naval Observatory, and the University of Washington.

The Millennium Simulation databases used in this paper and the web application providing online access to them were constructed as part of the activities of the German Astrophysical Virtual Observatory (GAVO).

The authors gratefully acknowledge the Gauss Centre for Supercomputing e.V. (www.gauss-centre.eu) and the Partnership for Advanced Supercomputing in Europe (PRACE, www.prace-ri.eu) for funding the MultiDark simulation project by providing computing time on the GCS Supercomputer SuperMUC at Leibniz Supercomputing Centre (LRZ, www.lrz.de). The Bolshoi simulations have been performed within the Bolshoi project of the University of California High-Performance AstroComputing Center (UCHiPACC) and were run at the NASA Ames Research Center.

\section{REFERENCES}

Abazajian K. N., et al., 2009, ApJS, 182, 543

Aihara H., et al., 2011, ApJS, 193, 29

Astropy Collaboration 2013, A\&A, 558, A33

Beers T. C., Flynn K., Gebhardt K., 1990, AJ, 100, 32

Behnel S., Bradshaw R., Citro C., Dalcin L., Seljebotn D. S., Smith K., 2011, Computing in Science \& Engineering, 13, 31 Behroozi P. S., Wechsler R. H., Wu H.-Y., 2013, ApJ, 762, 109

Bernardi M., Meert A., Sheth R. K., Vikram V., HuertasCompany M., Mei S., Shankar F., 2013, MNRAS, 436, 697

Binney J., Tremaine S., 1987, Galactic dynamics

Blanton M. R., Roweis S., 2007, AJ, 133, 734

Blanton M. R., et al., 2005, AJ, 129, 2562

Boylan-Kolchin M., Springel V., White S. D. M., Jenkins A., 2010, MNRAS, 406, 896

Buchner J., et al., 2014, A\&A, 564, A125

Cacciato M., van den Bosch F. C., More S., Li R., Mo H. J., Yang X., 2009, MNRAS, 394, 929

Cacciato M., van den Bosch F. C., More S., Mo H., Yang X., 2013, MNRAS, 430, 767

5 https://github.com/esheldon/pymangle
Campbell D., van den Bosch F. C., Hearin A., Padmanabhan N., Berlind A., Mo H. J., Tinker J., Yang X., 2015, MNRAS, 452, 444

Chen J., 2008, A\&A, 484, 347

Conroy C., Wechsler R. H., Kravtsov A. V., 2006, ApJ, 647, 201

Conroy C., et al., 2007, ApJ, 654, 153

Croton D. J., et al., 2006, MNRAS, 365, 11

Cui W., et al., 2016, MNRAS, 456, 2566

De Lucia G., Blaizot J., 2007, MNRAS, 375, 2

Diemand J., Moore B., Stadel J., 2004, MNRAS, 352, 535

Driver S. P., et al., 2011, MNRAS, 413, 971

Dutton A. A., Conroy C., van den Bosch F. C., Prada F., More S., 2010, MNRAS, 407, 2

Eisenstein D. J., et al., 2001, AJ, 122, 2267

Feroz F., Hobson M. P., 2008, MNRAS, 384, 449

Feroz F., Hobson M. P., Bridges M., 2009, MNRAS, 398, 1601

Feroz F., Hobson M. P., Cameron E., Pettitt A. N., 2013, preprint, (arXiv:1306.2144)

Foreman-Mackey D., 2016, The Journal of Open Source Software, 24

Guo Q., White S., Li C., Boylan-Kolchin M., 2010, MNRAS, 404, 1111

Guo Q., Cole S., Eke V., Frenk C., 2012, MNRAS, 427, 428

Guo Q., Cole S., Eke V., Frenk C., Helly J., 2013, MNRAS, 434, 1838

Guo H., et al., 2015a, MNRAS, 446, 578

Guo H., et al., 2015b, MNRAS, 453, 4368

Guo H., et al., 2016, MNRAS, 459, 3040

Hamilton A. J. S., Tegmark M., 2004, MNRAS, 349, 115

Hearin A. P., Zentner A. R., Newman J. A., Berlind A. A., 2013a, MNRAS, 430, 1238

Hearin A. P., Zentner A. R., Berlind A. A., Newman J. A., 2013b, MNRAS, 433, 659

Hearin A., et al., 2016, preprint, (arXiv:1606.04106)

Hikage C., Mandelbaum R., Takada M., Spergel D. N., 2013, MNRAS, 435, 2345

Hikage C., Mandelbaum R., Leauthaud A., Rozo E., Rykoff E. S., 2017, preprint, (arXiv:1702.08614)

Hoshino H., et al., 2015, MNRAS, 452, 998

Hunter J. D., 2007, Computing in Science and Engineering, 9, 90

Jiang F., van den Bosch F. C., 2017, MNRAS, 472, 657

Johnston D. E., et al., 2007, preprint, (arXiv:0709.1159)

Kauffmann G., Li C., Zhang W., Weinmann S., 2013, MNRAS, 430, 1447

Klypin A., Prada F., 2009, ApJ, 690, 1488

Klypin A., Yepes G., Gottlöber S., Prada F., Heß S., 2016, MNRAS, 457, 4340

Lauer T. R., Postman M., Strauss M. A., Graves G. J., Chisari N. E., 2014, ApJ, 797, 82

Leauthaud A., et al., 2012, ApJ, 744, 159

Li C., Jing Y. P., Mao S., Han J., Peng Q., Yang X., Mo H. J., van den Bosch F., 2012, ApJ, 758, 50

Li R., Mo H. J., Fan Z., Yang X., Bosch F. C. v. d., 2013, MNRAS, 430,3359

Li R., et al., 2014, MNRAS, 438, 2864

Li R., et al., 2016, MNRAS, 458, 2573

Lin Y.-T., Ostriker J. P., Miller C. J., 2010, ApJ, 715, 1486

Mao Y.-Y., Williamson M., Wechsler R. H., 2015, ApJ, 810, 21

Mo H., van den Bosch F. C., White S., 2010, Galaxy Formation and Evolution

Monaco P., Fontanot F., Taffoni G., 2007, MNRAS, 375, 1189

More S., 2012, ApJ, 761, 127

More S., van den Bosch F. C., Cacciato M., Mo H. J., Yang X., Li R., 2009a, MNRAS, 392, 801

More S., van den Bosch F. C., Cacciato M., 2009b, MNRAS, 392, 917

More S., van den Bosch F. C., Cacciato M., Skibba R., Mo H. J., Yang X., 2011, MNRAS, 410, 210 
Natarajan P., Kneib J.-P., 1997, MNRAS, 287, 833

Natarajan P., et al., 2017, MNRAS, 468, 1962

Navarro J. F., Frenk C. S., White S. D. M., 1996, ApJ, 462, 563

Niemiec A., et al., 2017, MNRAS, 471, 1153

Norberg P., Frenk C. S., Cole S., 2008, MNRAS, 383, 646

Oliva-Altamirano P., et al., 2014, MNRAS, 440, 762

Padmanabhan N., et al., 2008, ApJ, 674, 1217

Planck Collaboration et al., 2014, A\&A, 571, A16

Prada F., et al., 2003, ApJ, 598, 260

Riebe K., et al., 2011, preprint, (arXiv:1109.0003)

Robotham A. S. G., et al., 2011, MNRAS, 416, 2640

Sanderson A. J. R., Edge A. C., Smith G. P., 2009, MNRAS, 398, 1698

Sheldon E. S., et al., 2009a, ApJ, 703, 2217

Sheldon E. S., et al., 2009b, ApJ, 703, 2232

Shen S., Yang X., Mo H., van den Bosch F., More S., 2014, ApJ, 782,23

Sifón C., et al., 2015, MNRAS, 454, 3938

Skibba R. A., van den Bosch F. C., Yang X., More S., Mo H., Fontanot F., 2011, MNRAS, 410, 417

Spergel D. N., et al., 2007, ApJS, 170, 377

Springel V., et al., 2005, Nature, 435, 629

Swanson M. E. C., Tegmark M., Hamilton A. J. S., Hill J. C., 2008, MNRAS, 387, 1391

Tinker J. L., Hahn C., Mao Y.-Y., Wetzel A. R., Conroy C., 2017, preprint, (arXiv: 1702.01121)

Tremaine S. D., Richstone D. O., 1977, ApJ, 212, 311

Trevisan M., Mamon G. A., 2017, MNRAS, 471, 2022

Vale A., Ostriker J. P., 2004, MNRAS, 353, 189

Wang L., et al., 2014, MNRAS, 439, 611

Weinmann S. M., van den Bosch F. C., Yang X., Mo H. J., 2006, MNRAS, 366, 2

Wojtak R., Mamon G. A., 2013, MNRAS, 428, 2407

Yang X., Mo H. J., van den Bosch F. C., 2003, MNRAS, 339, 1057

Yang X., Mo H. J., van den Bosch F. C., Weinmann S. M., Li C., Jing Y. P., 2005, MNRAS, 362, 711

Yang X., Mo H. J., van den Bosch F. C., Jing Y. P., Weinmann S. M., Meneghetti M., 2006, MNRAS, 373, 1159

Yang X., Mo H. J., van den Bosch F. C., Pasquali A., Li C., Barden M., 2007, ApJ, 671, 153

Yang X., Mo H. J., van den Bosch F. C., 2008, ApJ, 676, 248

Ye J.-N., Guo H., Zheng Z., Zehavi I., 2017, ApJ, 841, 45

Zehavi I., et al., 2011, ApJ, 736, 59

Zentner A. R., Berlind A. A., Bullock J. S., Kravtsov A. V., Wechsler R. H., 2005, ApJ, 624, 505

Zentner A. R., Hearin A. P., van den Bosch F. C., 2014, MNRAS, 443, 3044

Zentner A. R., Hearin A., van den Bosch F. C., Lange J. U., Villarreal A., 2016, preprint, (arXiv:1606.07817)

Zheng Z., Coil A. L., Zehavi I., 2007, ApJ, 667, 760

Zitrin A., Bartelmann M., Umetsu K., Oguri M., Broadhurst T., 2012, MNRAS, 426, 2944

Zu Y., Mandelbaum R., Simet M., Rozo E., Rykoff E. S., 2017, MNRAS, 470, 551

van den Bosch F. C., Yang X., Mo H. J., 2003, MNRAS, 340, 771

van den Bosch F. C., Norberg P., Mo H. J., Yang X., 2004, MNRAS, 352, 1302

van den Bosch F. C., Tormen G., Giocoli C., 2005a, MNRAS, 359,1029

van den Bosch F. C., Weinmann S. M., Yang X., Mo H. J., Li C., Jing Y. P., 2005b, MNRAS, 361, 1203

van den Bosch F. C., Jiang F., Campbell D., Behroozi P., 2016, MNRAS, 455, 158

van der Walt S., Colbert S. C., Varoquaux G., 2011, Computing in Science \& Engineering, 13, 22

\section{APPENDIX A: CLF PREDICTIONS}

Here, we describe several relations regarding the probability of the BHG to be a satellite in CLF models. A common assumption in CLF modelling is that each halo hosts exactly one central (at least for the mass scale of interest here). This requires

$\int_{0}^{\infty} \Phi_{\mathrm{c}}\left(L_{\mathrm{c}} \mid M\right) d L_{\mathrm{c}}=1$.

Particularly, this implies that among those systems that do not have a central galaxy brighter than $L_{\max }$, their central $\operatorname{CLF} \Phi_{\mathrm{c}}^{\star}\left(L_{\mathrm{c}} \mid M, L_{\max }\right)$ must obey

$\int_{0}^{L_{\max }} \Phi_{\mathrm{c}}^{\star}\left(L_{\mathrm{c}} \mid M, L_{\max }\right) d L_{\mathrm{c}}=1$,

from which we find

$\Phi_{\mathrm{c}}^{\star}\left(L_{\mathrm{c}} \mid M, L_{\max }\right)=\frac{\Phi_{\mathrm{c}}\left(L_{\mathrm{c}} \mid M\right)}{\int_{0}^{L_{\max }} \Phi_{\mathrm{c}}\left(L_{\mathrm{c}} \mid M\right) d L_{\mathrm{c}}}$.

On the other hand, the occupation of satellites is assumed to follow a Poisson distribution and that luminosities of satellites are uncorrelated. This implies

$\Phi_{\mathrm{s}}^{\star}\left(L_{\mathrm{s}} \mid M, L_{\max }\right)=\Phi_{\mathrm{s}}\left(L_{\mathrm{s}} \mid M\right)$.

Now, given a halo with no central and satellite above a luminosity $L_{\mathrm{BHG}}$ but a galaxy with luminosity $L \in\left[L_{\mathrm{BHG}}-\right.$ $\left.d L, L_{\mathrm{BHG}}\right]$, the probability that this galaxy is a satellite is

$$
f_{\mathrm{BNC}}\left(L_{\mathrm{BHG}}, M\right)=\frac{\Phi_{\mathrm{s}}\left(L_{\mathrm{BHG}} \mid M\right)}{\Phi_{\mathrm{c}}^{\star}\left(L_{\mathrm{BHG}} \mid M, L_{\mathrm{BHG}}\right)+\Phi_{\mathrm{s}}\left(L_{\mathrm{BHG}} \mid M\right)},
$$

from which Eq. (12) follows.

The probability that a satellite brighter than some threshold luminosity $L_{\mathrm{th}}$ is brighter than the central with luminosity $L_{\mathrm{c}}$ is given by

$p\left(L_{\mathrm{s}}>L_{\mathrm{c}} \mid L_{\mathrm{s}}>L_{\mathrm{th}}\right)=\frac{\int_{L_{\mathrm{c}}}^{\infty} \Phi_{\mathrm{s}}\left(L_{\mathrm{s}} \mid M\right) d L_{\mathrm{s}}}{\int_{L_{\mathrm{th}}}^{\infty} \Phi_{\mathrm{s}}\left(L_{\mathrm{s}} \mid M\right) d L_{\mathrm{s}}}=\frac{\left\langle n_{\mathrm{BTC}}\right\rangle}{\left\langle n_{\mathrm{sat}}\right\rangle}$

Let us assume that the number of satellites fainter than $L_{\mathrm{c}}$ is fixed, $n_{\text {sat }}-n_{\mathrm{BTC}}=n_{\mathrm{FTC}}$. Then the probability to have $n_{\mathrm{BTC}}$ satellites brighter than $L_{\mathrm{c}}$ is

$$
\begin{aligned}
p\left(n_{\mathrm{BTC}} \mid n_{\mathrm{FTC}}\right)= & p\left(n_{\mathrm{sat}}=n_{\mathrm{FTC}}+n_{\mathrm{BTC}}\right) \times \\
& p\left(n_{\mathrm{BTC}} \mid n_{\mathrm{sat}}=n_{\mathrm{FTC}}+n_{\mathrm{BTC}}\right) \\
\propto & \frac{\left\langle n_{\mathrm{sat}}\right\rangle^{n_{\mathrm{BTC}}}}{\left(n_{\mathrm{FTC}}+n_{\mathrm{BTC}}\right) !}\left(\begin{array}{c}
n_{\mathrm{FTC}}+n_{\mathrm{BTC}} \\
n_{\mathrm{BTC}}
\end{array}\right) \times \\
& \left(\frac{\left\langle n_{\mathrm{BTC}}\right\rangle}{\left\langle n_{\mathrm{sat}}\right\rangle}\right)^{n_{\mathrm{BTC}}} \\
\propto & \frac{\left\langle n_{\mathrm{BTC}}\right\rangle^{n_{\mathrm{BTC}}}}{n_{\mathrm{BTC}} !} .
\end{aligned}
$$

That means that $n_{\mathrm{BTC}}$ also follows a Poisson distribution and is completely independent of the occupation with satellites fainter than $L_{\mathrm{c}}$. From this, we can easily show that the 
probability that any satellite is brighter than the central luminosity $L_{\mathrm{c}}$ is

$$
\begin{aligned}
f_{\mathrm{BNC}} & =\sum_{n_{\mathrm{BTC}}=1}^{\infty} \frac{\left\langle n_{\mathrm{BTC}}\right\rangle^{n_{\mathrm{BTC}}}}{n_{\mathrm{BTC}} !} \exp \left(-\left\langle n_{\mathrm{BTC}}\right\rangle\right) \\
& =1-\exp \left(-\left\langle n_{\mathrm{BTC}}\right\rangle\right) .
\end{aligned}
$$

Finally, the probability that a halo of mass $M$ has no galaxy above luminosity $L_{\max }$ is

$$
\begin{aligned}
p\left(L_{\mathrm{BHG}} \leqslant L_{\max }\right)= & \left(\int_{0}^{L_{\max }} \Phi_{\mathrm{c}}\left(L_{\mathrm{c}} \mid M\right) d L_{\mathrm{c}}\right) \times \\
& \exp \left[-\int_{L_{\max }}^{\infty} \Phi_{\mathrm{s}}\left(L_{\mathrm{s}} \mid M\right) d L_{\mathrm{s}}\right] .
\end{aligned}
$$

The first term in the right-hand side of the above equation describes the probability that the central is not brighter than $L_{\max }$, whereas the second term describes the probability that no satellite galaxy is brighter than $L_{\max }$. Ultimately, the CLF of the BHG follows

$\Phi_{\mathrm{BHG}}(L \mid M)=p\left(L \leqslant L_{\mathrm{BHG}}\right)\left(\Phi_{\mathrm{c}}^{\star}\left(L \mid M, L_{\mathrm{BHG}}\right)+\Phi_{\mathrm{s}}(L \mid M)\right)$

$$
\begin{aligned}
= & {\left[\Phi_{\mathrm{c}}(L \mid M)+\Phi_{\mathrm{s}}(L \mid M) \int_{0}^{L_{\mathrm{BHG}}} \Phi_{\mathrm{c}}\left(L_{\mathrm{c}} \mid M\right) d L_{\mathrm{c}}\right] } \\
& \times \exp \left[-\int_{L_{\mathrm{BHG}}}^{\infty} \Phi_{\mathrm{s}}\left(L_{\mathrm{s}} \mid M\right) d L_{\mathrm{s}}\right] .
\end{aligned}
$$

